ARTICLE

httpst//doi.org/10.1038/s41467-020-17126-3

\title{
Highly reversible oxygen redox in layered compounds enabled by surface polyanions
}

\author{
Qing Chen ${ }^{1,2,7}$, Yi Pei ${ }^{1,7}$, Houwen Chen ${ }^{3}$, Yan Song (i) ${ }^{4}$, Liang Zhen (1) ${ }^{1,5}$, Cheng-Yan Xu (i) ${ }^{1,5 凶}$, \\ Penghao Xiao (10 ${ }^{2,6 \bowtie} \&$ Graeme Henkelman (10) ${ }^{2 凶}$
}

Oxygen-anion redox in lithium-rich layered oxides can boost the capacity of lithium-ion battery cathodes. However, the over-oxidation of oxygen at highly charged states aggravates irreversible structure changes and deteriorates cycle performance. Here, we investigate the mechanism of surface degradation caused by oxygen oxidation and the kinetics of surface reconstruction. Considering $\mathrm{Li}_{2} \mathrm{MnO}_{3}$, we show through density functional theory calculations that a high energy orbital $\left(\mathrm{IO}_{2 p^{\prime}}\right)$ at under-coordinated surface oxygen prefers over-oxidation over bulk oxygen, and that surface oxygen release is then kinetically favored during charging. We use a simple strategy of turning under-coordinated surface oxygen into polyanionic $\left(\mathrm{SO}_{4}\right)^{2-}$, and show that these groups stabilize the surface of $\mathrm{Li}_{2} \mathrm{MnO}_{3}$ by depressing gas release and side reactions with the electrolyte. Experimental validation on $\mathrm{Li}_{1.2} \mathrm{Ni}_{0.2} \mathrm{Mn}_{0.6} \mathrm{O}_{2}$ shows that sulfur deposition enhances stability of the cathode with $99.0 \%$ capacity remaining (194 $\mathrm{mA} \mathrm{h}^{-1}$ ) after 100 cycles at $1 \mathrm{C}$. Our work reveals a promising surface treatment to address the instability of highly charged layered cathode materials.

\footnotetext{
${ }^{1}$ School of Materials Science and Engineering, and MOE Key Laboratory of Micro-Systems and Micro-Structures Manufacturing, Harbin Institute of Technology, Harbin 150001, China. ${ }^{2}$ Department of Chemistry and the Oden Institute for Computational Engineering and Sciences, The University of Texas at Austin, Austin, TX 78712-0165, USA. ${ }^{3}$ College of Materials Science and Engineering, Chongqing University, Chongqing 400044 , China. ${ }^{4}$ School of Materials Science and Engineering, Harbin Institute of Technology (Weihai), Weihai 264209, China. ${ }^{5}$ School of Materials Science and Engineering, Harbin Institute of Technology (Shenzhen), Shenzhen 518055, China. ${ }^{6}$ Materials Science Division, Lawrence Livermore National Laboratory, Livermore, CA 94550, USA. ${ }^{7}$ These authors contributed equally: Qing Chen, Yi Pei. ${ }^{凶}$ email: cy_xu@hit.edu.cn; xiao2@llnl.gov; henkelman@utexas.edu
} 
$\mathrm{n}$ the past few decades, Li-ion batteries with high energy density have been intensively investigated due to the increasing requirements of the emerging market of mobile devices and electric vehicles. Cathode materials, including Li-rich NMC $\left(\mathrm{Li}_{1.2} \mathrm{Ni}_{0.13} \mathrm{Mn}_{0.54} \mathrm{Co}_{0.13} \mathrm{O}_{2}\right)$ with high capacity and energy density, have thus attracted tremendous attention. At present, the capacity of conventional cathode materials is largely restricted by the limited capability of removable $\mathrm{Li}^{+}$ions, which are chargecompensated by cationic redox, so that the practical capacity has approached a limit of about $200 \mathrm{~mA} \mathrm{~h} \mathrm{~g}^{-1}$. Recently, a new anion redox couple, the reversible $\mathrm{O}^{2-} /\left(\mathrm{O}_{2}\right)^{n-}$ redox reaction, has been widely understood to take place in lithium-rich layered oxides (LLO), which offers a new paradigm to boost the capacity of cathode materials up to $\sim 300 \mathrm{~mA} \mathrm{~h}^{-1}$.

The chemical nature of oxygen with redox activity in LLO has been studied theoretically. Based on observations of the local environments of $\mathrm{O}$ ions in LLO, it was found that the $\mathrm{O}-2 p$ orbital along the $\mathrm{Li}-\mathrm{O}-\mathrm{Li}$ direction will generate a specific nonbonding band with a higher energy level ${ }^{1}$. These nonbonding orbitals will be oxidized preferentially during the delithiation process and thus contribute to the extra capacity of LLO. It has been further understood that a reversible oxygen redox can be achieved only when the antibonding transition-metal (TM)-O band of $\mathrm{TMO}_{6}$ ligands partially overlaps with the O-2p nonbonding band or with the antibonding orbital of $\mathrm{O}-\mathrm{O}$ dimers ${ }^{2,3}$. However, for earth-abundant $3 d-\mathrm{TM}$ ions, the insufficient covalency of TM-O band prohibits charge transfer between TM and $\mathrm{O}^{2-}$, resulting in an undesirable irreversible oxygen oxidation ${ }^{4,5}$ and subsequent structural degradation and detrimental electrochemical performance ${ }^{6,7}$. This chemical picture accounts for the observation of gaseous oxygen evolution in the first cycle by in situ differential electrochemical mass spectrometry ${ }^{8}$. Recently, an alternative redox mechanism in Li-excess manganese oxides was proposed ${ }^{9}$; a small fraction of $\mathrm{Mn}^{4+}$ will be oxidized to $\mathrm{Mn}^{7+}$ followed by the spontaneous dimerization of oxygen during charge. The multiple redox process causes the voltage hysteresis and voltage fade.

Until now, most theoretical research has focused on the thermodynamics of oxygen redox, and the inevitable thermodynamic instability at highly charged states has been confirmed by calculating the enthalpy of the oxygen loss reaction, especially in $3 d$ TM containing LLOs ${ }^{10,11}$. However, even though the peroxide dimer or a trapped oxygen molecule could form inside the material ${ }^{9,12,13}$, gaseous oxygen release from the bulk is kinetically unlikely ${ }^{14,15}$, due to kinetic prohibition for oxygen interstitial migration ${ }^{16}$. This explains previous experiment results showing that the oxygen in bulk LLO can undergo reversible redox and contribute to the reversible capacity ${ }^{17-20}$. Therefore, the kinetics of oxygen loss is essential to understand the irreversible capacity loss during cycling in LLO. To optimize the electrochemical performance of LLOs, it is critical to mitigate surface oxygen release and thus kinetically impede oxygen loss in the entire material while not affecting oxygen redox in the bulk material. As long as $\mathrm{O}$ is retained in the structure, other structural changes are likely reversible upon discharging ${ }^{21}$. Various surface modifications have therefore been proposed to suppress the irreversible process, and substantial improvements have been achieved ${ }^{22-26}$. Nevertheless, a fundamental understanding of surface oxygen evolution, including energetics and kinetics, is still missing, and this is essential to understanding the reversibility of anion redox and the metastable nature of highly charged layered materials.

In this work, we theoretically investigate the surface oxygen on $\mathrm{Li}_{2} \mathrm{MnO}_{3}$, which is the end member and primary component after the "activation" of LLO. The overoxidation of surface oxygen is attributed to its undercoordinated configuration with cationic vacancies on the surface, which creates an additional higher energy orbital $\left(\mathrm{l}_{2 p^{\prime}}\right)$. The calculated minimum energy path (MEP) shows that the kinetically favored surface oxygen release gives rise to surface $\mathrm{Mn}$ back diffusion and thus triggers a spinellike phase transformation. We therefore induce surface polyanion formation by sulfur deposition to avoid the nonbonding coordination of surface oxygen and thus stabilize the surface. These theoretical predictions are confirmed by experimental cycling tests carried out in a practical $\mathrm{LLO}, \mathrm{Li}_{1.2} \mathrm{Ni}_{0.2} \mathrm{Mn}_{0.6} \mathrm{O}_{2}$. This study uncovers a promising path toward addressing the issue of cycled structure instability in current cathode materials that resulted from surface anionic oxidation.

\section{Results}

Surface oxygen oxidation and correlation with surface structural transformation in $\mathrm{Li}_{2} \mathbf{M n O}_{3}$. To study the surface oxygen evolution, three low-index surfaces of $\mathrm{Li}_{2} \mathrm{MnO}_{3}$, i.e., (001), (010), and (110), were examined. These surfaces are considered to be stable and electrochemically active ${ }^{27}$. Various terminations and their corresponding surface Li-binding energies were examined (Supplementary Fig. 1), revealing that at the beginning of charge, the most stable (001) surface is terminated by $\mathrm{O}$ ions, and the stable $(010) /(110)$ surfaces are terminated by Li ions. To distinguish the oxygen oxidation in surface and bulk, we studied $\mathrm{O}$ ions at different layers from the outermost layer to the innermost, as shown for the (010) surface in Fig. 1a (the other two surfaces are shown in Supplementary Fig. 2). The redox activity of oxygen atoms from the first layer to the fourth layer in the three surfaces was predicted by the calculated local density of states (LDOS) using the HSE06 hybrid functional ${ }^{28}$ (Fig. 1b). It is shown that the bands near the Fermi level are mostly from the oxygen ions in the outermost layer. Here, the $\mathrm{O}$ ions in the first layer are denoted as $\mathrm{O}_{\text {sur, }}$, while in the other layers, are considered as $\mathrm{O}_{\text {int }}$. It can then be concluded that the $2 p$ orbital of $\mathrm{O}_{\text {sur }}$ has a higher energy level than that of $\mathrm{O}_{\text {int }}$, suggesting a preferred oxidation of $\mathrm{O}_{\text {sur }}$ in the delithiation process.

Prior to investigating oxygen reactions on the surface, the electrochemical activity of each surface was examined by evaluating its Li transfer ability. We calculated the preferred diffusion paths of $\mathrm{Li}$ extraction from each surface and the corresponding kinetic barriers (Supplementary Fig. 3), showing that the rate-limiting mechanisms of sequential diffusion steps are similar, and thus the three surfaces can delithiate spontaneously. To simulate a practical degree of $\mathrm{O}^{2-}$ oxidation in $\mathrm{Li}$-rich layered oxides $\left(0.7-0.8 \mathrm{e}^{-} \text {per formula unit }\right)^{8,29}$, the delithiated state on each surface was set as $37.5 \%\left(0.75 \mathrm{e}^{-}\right.$formally extracted from $\mathrm{O}$ per formula unit). The basin-hopping global optimization algorithm was then utilized to search for the lowest energy structure in each surface, to find that $\mathrm{Li}$ vacancies are primarily distributed in the outermost part of the slab (Supplementary Fig. 4). This is in agreement with a previous observation from electron energy loss spectroscopy (EELS), which indicated that the concentration of $\mathrm{Li}$ in the surface is less than that in the interior area, even when the cell is fully discharged ${ }^{30}$. As a result of the inhomogeneous distribution of $\mathrm{Li}$ vacancies, three pairs of $\mathrm{O}_{\text {sur }}$ rotate to spontaneously form $\mathrm{O}_{\text {sur }}-\mathrm{O}_{\text {sur }}$ dimers on the (010) and (110) surfaces at the $37.5 \%$ delithiation state, whereas no $\mathrm{O}_{\text {int }}-\mathrm{O}_{\text {int }}$ formation is seen in the slabs, indicating a higher oxidation state of $\mathrm{O}_{\text {sur. }}$. For the (001) surface, the $\mathrm{O}-\mathrm{O}$ dimers also form only at the surface with a low barrier $(0.2 \mathrm{eV})$, consistent with a higher oxidation state of $\mathrm{O}_{\text {sur; }}$; details of this mechanism will be discussed later.

To quantify the charge state of $\mathrm{O}_{\text {sur }}$ and $\mathrm{O}_{\text {int }}$ during delithiation, we calculated the Bader charge (per atom) of $\mathrm{O}$ and $\mathrm{Mn}$ atoms in each surface at two delithiated states (Fig. 1c). A negative value for the change of Bader charge indicates electron 
a

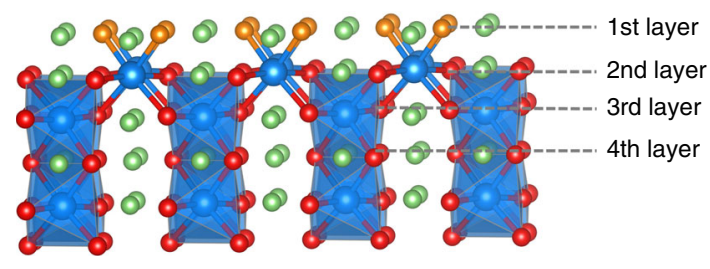

b

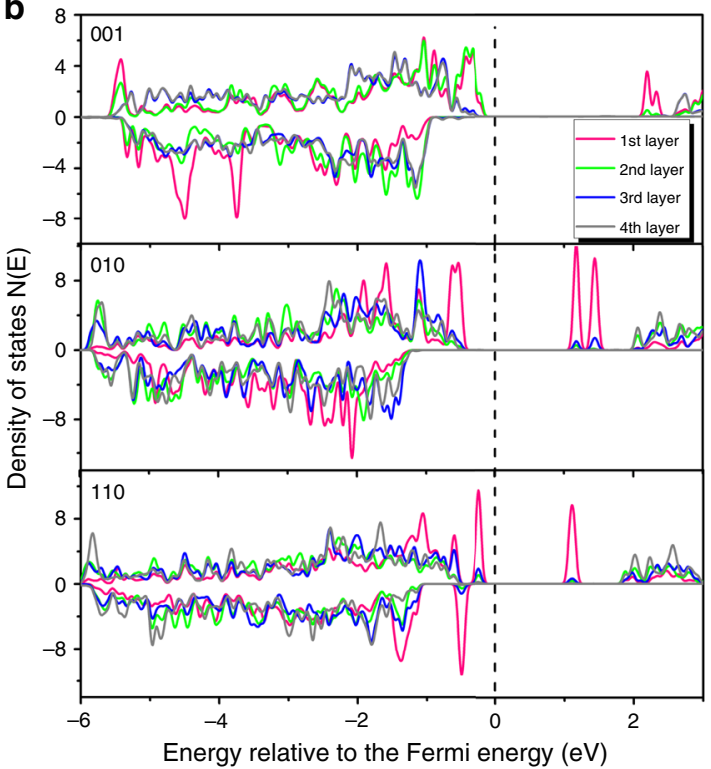

C

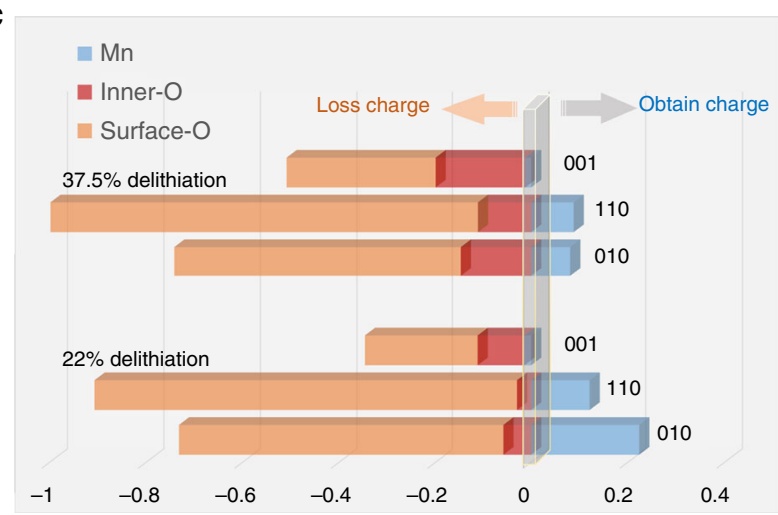

d

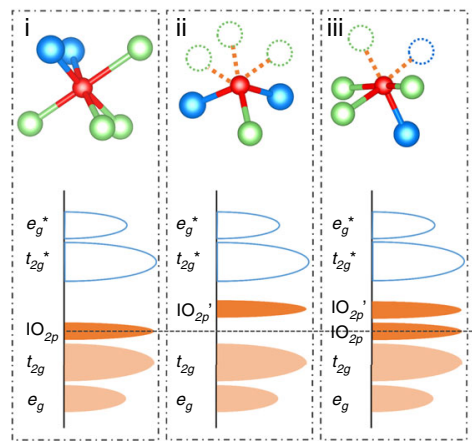

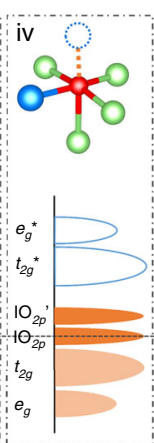

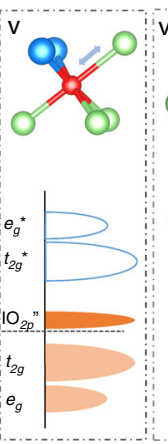

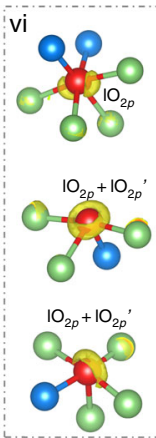

Fig. 1 Redox mechanism on the surface of $\mathrm{Li}_{2} \mathbf{M n O}_{\mathbf{3}}$ a Crystal structure of $\mathrm{Li}_{2} \mathrm{MnO}_{3}(010)$ surface. The oxygen ions are divided into four layers from the outmost surface to the inner; each layer has the same number of $\mathrm{O}$ ions. The blue, green, and red/golden balls represent $\mathrm{Mn}$, Li, and $\mathrm{O}$ ions, respectively. b Calculated LDOS of oxygen ions from the first layer to the fourth layer in the (001), (010), and (110) surfaces, respectively. The Fermi level is indicated by the dashed line. $\mathbf{c}$ Bader analysis of the surface $\mathrm{O}$, inner $\mathrm{O}$, and $\mathrm{Mn}$ ions of the three slabs in different delithiation states. Negative values of the change of Bader charge indicate electron loss of the ions. d Schematic diagram of various local atomic configurations, as well as the electronic structures and the calculated ELF. i, $\mathrm{O}_{\text {int, }}$ ii, $\mathrm{O}_{\text {sur }}$ on the (001) surface, iii, $\mathrm{O}_{\text {sur }}$ on the (010) surface and partial $\mathrm{O}_{\text {sur }}$ on the (110) surface, iv, partial $\mathrm{O}_{\text {sur }}$ on the (110) surface, $\mathrm{v}$, oxygen ions in the second layer of the (001) surface, and vi, ELF values computed (isovalues of 0.7) for $\mathrm{O}_{\text {int }}$ and $\mathrm{O}_{\text {sur }}$ in iii and iv. The hollow blue area, filled orange area, and filled light-orange area in each electronic state represents the empty antibonding $(\mathrm{M}-\mathrm{O})^{\star}$ band, $\mathrm{O}(2 p)$ lone-pair band, and bonding $(\mathrm{M}-\mathrm{O})$ band, respectively.

loss at the ions, corresponding to the oxidation reaction. As revealed by the Bader analysis, charge compensation of the whole system is dominated by the oxidation of $\mathrm{O}_{\text {sur }}$ with more electron loss, although the oxidation of $\mathrm{O}_{\text {int }}$ is enhanced with the state of delithiation from around $22 \%$ to $37.5 \%$; the slight reduction of $\mathrm{Mn}$ suggests charge transfer between $\mathrm{O}$ and $\mathrm{Mn}$. The higher oxidation of $\mathrm{O}_{\text {sur }}$ than $\mathrm{O}_{\text {int }}$ during delithiation is not surprising because the Li vacancies are prone to aggregation at the surface, which requires more charge compensation.

In essence, the prior oxidation of $\mathrm{O}_{\text {sur }}$ can be understood by the specific surface-coordinated configuration at the electronic structure level. In contrast with $\mathrm{O}_{\text {int }}$ that has one $\mathrm{lO}_{2 p}$ pair (an $\mathrm{O}(2 p)$ lone pair) ${ }^{31}$ ( $\mathrm{i}$ in Fig. 1d), the local coordination of $\mathrm{O}_{\text {sur }}$ in our models can be used to categorize $\mathrm{O}_{\text {sur }}$ into three species with different cationic vacancies (ii, iii, and iv in Fig. 1d). These surface cationic vacancies result in a reduced bonding of $\mathrm{O}_{\text {sur }}$ along the $\square-\mathrm{O}_{\text {sur }}$ - Li direction ( $\square$ represents cationic vacancies) with respect to that of $1_{2 p}$ in $\mathrm{O}_{\text {int }}$. The $\square-\mathrm{O}_{\text {sur }}$ - $\mathrm{Li}$ configuration on the surface therefore generates an $\mathrm{lO}_{2 p}$, orbital with higher energy level than $\mathrm{lO}_{2 p}$, which can be seen in the higher energy level of $\mathrm{O}$ in the first layer, as shown in the LDOS (Fig. 1b). The increased number of lone-pair orbitals (iii and iv in Fig. 1d) can been directly observed in the electron localization function (ELF) ${ }^{31,32}$ results (vi in Fig. 1d). It should be mentioned that the oxygen ions in the second layer of (001) surface have a comparable energy level with that of the first layer, which is probably a result of the elongated $\mathrm{Li}-\mathrm{O}$ distance in the second layer that raises the energy level of $1_{2 p}$ to $\mathrm{lO}_{2 p}$ " ( $\mathrm{v}$ in Fig.1d).

The kinetic mechanism of irreversible oxygen release and the subsequent structural transformation were investigated to directly correlate the oxygen oxidation with $\mathrm{O}_{2}$ gas release and surface reconstruction. We calculated the minimum energy path (MEP) of the structural evolution on the (001) and (010) surfaces upon $37.5 \%$ delithiation (Fig. 2). The kinetic barriers of irreversible $\mathrm{O}_{2}$ release from the (001) and (010) surfaces are 0.60 and $0.66 \mathrm{eV}$, respectively, implying that the reaction of $\mathrm{O}_{2}$ released from the crystal is kinetically feasible at room temperature. Here, the concentration of oxygen vacancies after $\mathrm{O}_{2}$ evolution in the first layer is $33 \%$ in the (001) slab and $25 \%$ in the (010) slab. The onset of $\mathrm{O}_{2}$ evolution at these surfaces is expected with activation barriers no greater than 0.60 and $0.66 \mathrm{eV}$. The surface $\mathrm{O}_{2}$ release barrier will increase with the presence of oxygen vacancies and Mn densification near the surface, which explains why the irreversible oxygen release occurs primarily during the first charging cycle. We also utilized strongly constrained and appropriately normed density functional (SCAN) ${ }^{33,34}$, which is more accurate for the calculation of gas-phase oxygen, to obtain the MEP for lattice oxygen evolution to $\mathrm{O}_{2}$ on both surfaces (Supplementary Fig. 5), further revealing the kinetically feasible barriers $(<0.60 \mathrm{eV})$ for $\mathrm{O}_{2}$ release. As mentioned previously, the 
a
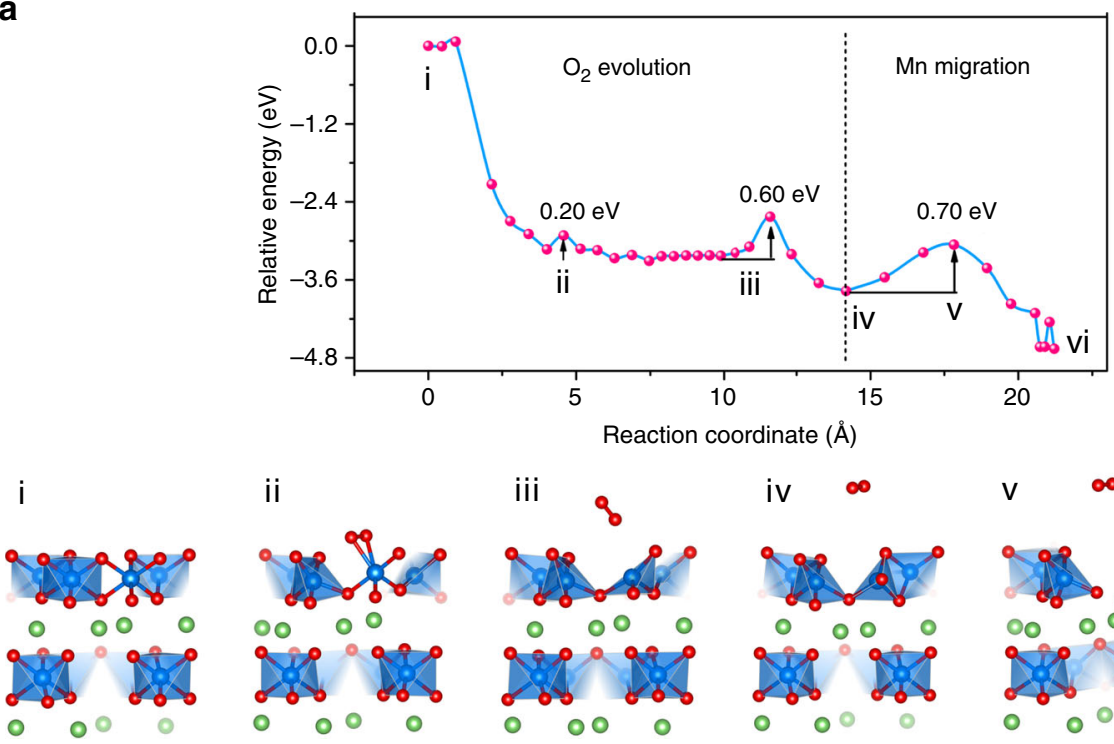

ii

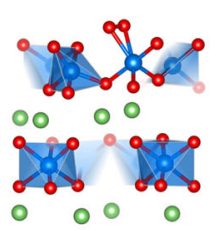

iii

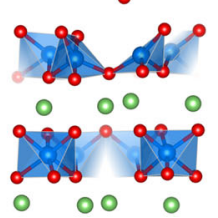

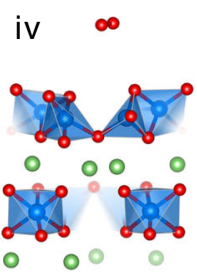
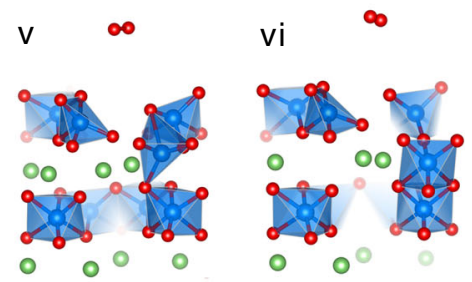

b
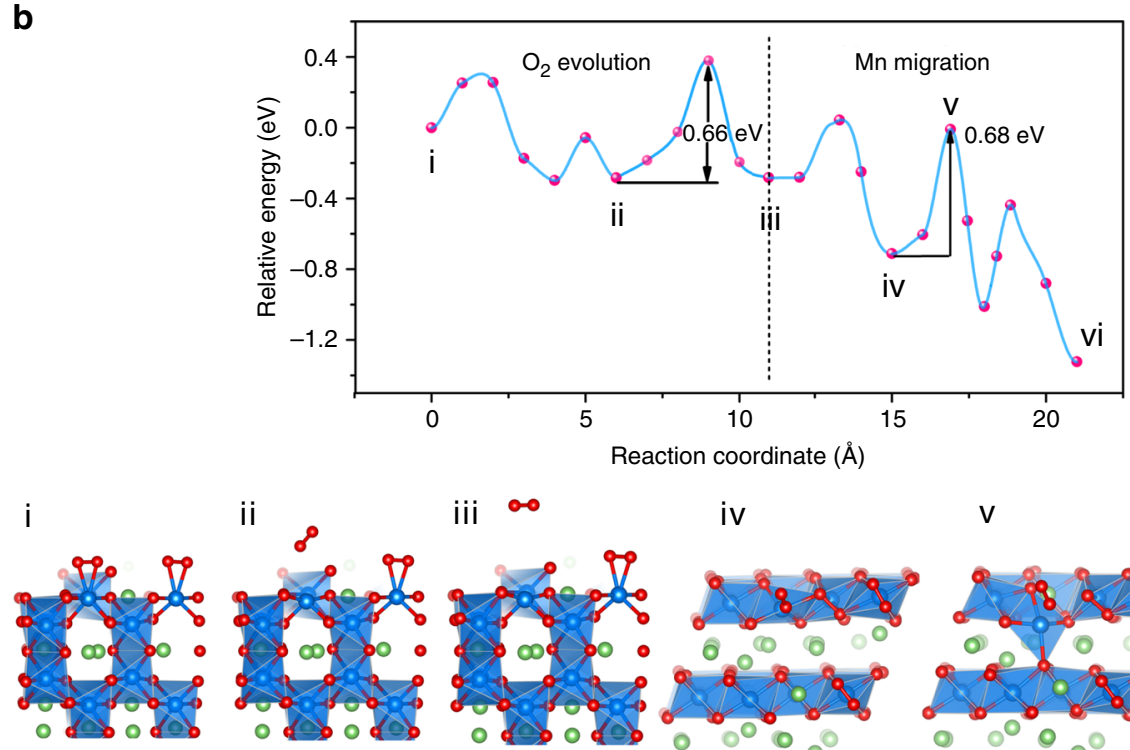

iv
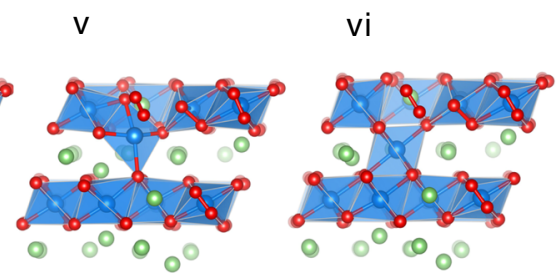

Fig. 2 Minimum energy path (MEP) plot for $\mathbf{O}_{\mathbf{2}}$ evolution and $\mathbf{M n}$ migration at the surface of $\mathbf{L i}_{\mathbf{2}} \mathbf{M n n} \mathbf{O}_{\mathbf{3}}$. $\mathbf{a}, \mathbf{b} \mathrm{MEP}$ plot for $\mathrm{O}_{2}$ evolution and $\mathrm{Mn}$ migration from the TM layer to the Li layer (upper) in the (001) (a) and (010) (b) surfaces, and the corresponding structures of some intermediates and transition states (below). All slabs in (a) and the first three slabs in (b) are shown as side views, while the last three slabs in (b) are top views to better visualize the Mn migration pathway.

facile oxidation of $\mathrm{O}_{\text {sur }}$ in $\mathrm{Li}_{2} \mathrm{MnO}_{3}$ is due to its reduced bonding coordination. Therefore, it should be possible to increase the $\mathrm{O}_{2}$ evolution barrier to mitigate or delay the irreversible $\mathrm{O}_{2}$ release by introducing electron donor.

With the surface oxygen dimer separated from $\mathrm{Mn}$, the $\mathrm{MnO}_{6}$ octahedron changes to $\mathrm{MnO}_{4}$ tetrahedron and the $\mathrm{Mn}$ moves to a more stable environment. From the calculated MEP plot of Mn migration followed by oxygen release on both surfaces (Fig. 2), the final states of the $\mathrm{MnO}_{6}$ octahedron located in the $\mathrm{Li}$ layer are more stable than the initial states of the $\mathrm{MnO}_{4}$ tetrahedron in the TM layer. During the process of $\mathrm{Mn}$ migration, a regular $\mathrm{MnO}_{4}$ tetrahedron is formed spontaneously (on the (001) surface) or with a small barrier (on the (010) surface) after oxygen release, while the subsequent migration of $\mathrm{Mn}$ ions into nearby interstitial octahedral sites in the Li layer is a slow step with a barrier of $0.7 \mathrm{eV}$. Meanwhile, Mn migration promotes the rearrangement of $\mathrm{Li}$ ions from interstitial octahedral sites to tetrahedral sites, thus forming a local spinel-like structure. The calculated surface kinetic process provides theoretical support for the experimentally observed structural transformation from a layered to spinellike or rock-salt phase in cycled LLO. In addition, the high reverse barrier for $\mathrm{Mn}$ migration back to the initial position during discharge accounts for the deterioration of electrochemical reversibility.

From the above discussion, the specific coordination configuration of $\mathrm{O}_{\text {sur }}$ changes its electronic structure by introducing an additional $1 \mathrm{O}_{2 p}$, band with a higher energy level than $1 \mathrm{O}_{2 p}$, inducing the preferred oxidation of $\mathrm{O}_{\text {sur. }}$. In addition, the calculated kinetic barrier of $\mathrm{O}_{\text {sur }}$ release $(\leq 0.66 \mathrm{eV})$ is accessible at room temperature. The kinetically feasible oxygen release then leads to a surface structural reconstruction via Mn migration. At this point, the reverse process in the discharged state, which requires $\mathrm{O}_{2}$ dissociation and $\mathrm{Mn}$ extraction, becomes kinetically prohibitive. We did not calculate the barrier for the reverse 
process, but it is well known that $\mathrm{O}_{2}$ dissociation on oxides is kinetically hindered at room temperature ${ }^{35,36}$. Consequently, $\mathrm{O}_{2}$ release is the detrimental factor for reversibility. Strategies that target manipulation of the electronic structure of $\mathrm{O}_{\text {sur }}$ and $\mathrm{O}_{2}$ evolution barrier are thus promising routes to mitigate $\mathrm{O}_{2}$ release and retain the reversibility of layered compounds.

Surface polyanion induction effect stabilizes surface oxygen. Since the overoxidation of $\mathrm{O}_{\text {sur }}$ stems from a deficiency of cationic bonding on the surface, we aim to reintroduce cationic species into the surface to bond with $\mathrm{O}_{\text {sur }}$, with the expectation of reducing nonbonding coordination. Inspired by the achievement of stabilizing oxygen through the inductive effect in polyanion compounds $^{37}$, surface deposition of less electronegative sulfur was utilized to stabilize $\mathrm{O}_{\text {sur }}$ in $\mathrm{Li}_{2} \mathrm{MnO}_{3}$. Sulfur deposition was carried out on three surfaces of $\mathrm{Li}_{2} \mathrm{MnO}_{3}$. The deposition energy at different sites and with different sulfur concentrations is compared in Supplementary Fig. 6. The results show that sulfitelike species $\left(\mathrm{SO}_{3}\right)^{x-}$ form on the $(001)$ surface and $\left(\mathrm{SO}_{2}\right)^{y-}$ forms on the (010) and (110) surfaces; these surface oxysulfides were confirmed to be thermodynamically stable. The formation of $\left(\mathrm{SO}_{n}\right)^{m-}$ polyanions suggests that electrons transfer from the less electronegative sulfur to $\mathrm{O}_{\text {sur }}$ by the inductive effect. Li-ion mobility in the sulfur-deposited structure was evaluated by calculating the preferred diffusion paths and the migration barriers (Supplementary Fig. 7). The calculated diffusion barriers (between 0.71 and $0.86 \mathrm{eV}$ ) in all the three surfaces are comparable with those before sulfur deposition (between 0.74 and $0.79 \mathrm{eV}$ ), indicating that the sulfur deposition does not hinder transport of Li from the surface.

After extraction of $37.5 \% \mathrm{Li}$, no $\mathrm{O}-\mathrm{O}$ dimers are observed on the sulfur-deposited surfaces; instead, the $\left(\mathrm{SO}_{3}\right)^{x-}$ and $\left(\mathrm{SO}_{2}\right)^{y-}$ species are oxidized to sulfate species $\left(\mathrm{SO}_{4}\right)^{2-}$ with tetrahedral configurations (Fig. 3a). This indicates that the redox center on the sulfur-deposited surfaces has shifted from $\mathrm{O}^{2-} / \mathrm{O}^{-}$to $\left(\mathrm{SO}_{n}\right)^{m-} /\left(\mathrm{SO}_{4}\right)^{2-}$, that is, sulfur ions provide the charge compensation on the surface in the first delithiation step. The top view of each surface after $37.5 \%$ delithiation (Supplementary Fig. 8) shows that all the $\mathrm{O}_{\text {sur }}$ has been coordinated with sulfide ions, indicating that the undesirable nonbonding coordination of $\mathrm{O}_{\text {sur }}$ can be effectively avoided by sulfur deposition. Note that the two outermost $\mathrm{Mn}$ ions on the (001) surface spontaneously migrate into Li-vacancy sites in the Li layer to form $\mathrm{MnO}_{6}$ octahedra after $\mathrm{Li}$ extraction, while no $\mathrm{Mn}$ migration was observed on the (010) and (110) surfaces. This can be attributed to the different orientations of the repulsive interaction between the high-valency $\mathrm{S}$ and $\mathrm{Mn}$ ions in the different surfaces. Nevertheless, the reconstructed surfaces reveal that sulfur deposition will likely promote the formation of $\mathrm{Li} / \mathrm{Mn}$ antisite mixing during delithiation. The delithiation voltages of the three structures (Fig. 3a) are calculated to be $2.97,3.05$, and $2.86 \mathrm{eV}$, respectively. These low voltages suggest that the delithiated states are stable, i.e., there is facile formation of the reconstructed surfaces.

To determine the stability of $\mathrm{O}_{\text {sur }}$ after sulfur deposition during charging, we calculated the energetics of all possible oxygen evolution mechanisms on all three surfaces, to release $\mathrm{O}_{2}, \mathrm{SO}_{2}$, or $\mathrm{SO}_{3}$. To simplify the description of our results, we labeled the $\mathrm{O}_{\text {sur }}$ in each surface, and assigned it to various possible products in the gas phase $\left(\mathrm{O}_{2}, \mathrm{SO}_{2}\right.$, and $\left.\mathrm{SO}_{3}\right)$ as the final states (see Fig. $3 \mathrm{~b}$ ). The energies of these final states are at least $1.2 \mathrm{eV}$ higher than the initial states, indicating that $\mathrm{O}_{\text {sur }}$ in the form of polyanions are energetically stable against gas evolution. We then utilized the more accurate SCAN functional to calculate the energy difference on (001) surface, to spot-check the possibility of oxygen loss. The calculated energy differences are all greater than $2.8 \mathrm{eV}$, showing even higher stability than the $\mathrm{PBE}+\mathrm{U}$ results. Surface oxygen stabilization is expected to enhance the reversible oxygen redox and promote capacity retention.

In addition, we consider the (010) surface as representative to check the surface stability under more practical electrolyte conditions. The gas evolution behavior in the presence of solvent was investigated using an implicit solvent model as implemented in the VASPsol package ${ }^{38}$. The reaction energy of all possible $\mathrm{O}_{\text {sur }}$ evolution with solvent dielectric constants of 30,60 , and $90\left(\varepsilon_{0}=\right.$ 2 in dimethyl carbonate (DMC), $\varepsilon_{0}=90$ in ethylene carbonate $(\mathrm{EC}))^{39}$ is calculated to be greater than $3.0 \mathrm{eV}$ (Fig. 3c), suggesting that the polyanionic $\left(\mathrm{SO}_{4}\right)^{2-}$ is stable with or without the solvation effect. Reaction with the electrolyte was also investigated. Referring to previous reports on the reaction between $\mathrm{Li}$ transition-metal oxides and conventional electrolytes, EC has been found to preferentially adsorb and react with the electrodes $^{40,41}$. In general, EC reaction/decomposition initiates by breaking one of the two $\mathrm{C}-\mathrm{O}$ bond in the ring on the cathode surfaces ${ }^{42,43}$, and finally evolves to $\mathrm{CO}_{2}$ and various other organic species $^{44,45}$. Therefore, our calculations focus on the first step of EC decomposition, the ring opening of cyclic carbonate after EC molecular adsorption on the delithiated (010) surface, as shown in Fig. 3d. The reaction energy was calculated to be -2.67 on the pristine surface and $1.53 \mathrm{eV}$ on the S-deposited surface using the SCAN functional, indicating that the onset of EC degradation is exothermic on the S-free surface of delithiated $\mathrm{Li}_{2} \mathrm{MnO}_{3}$, but energetically prohibitive after forming the polyanionic configuration. This is in agreement with previous reports that surface oxygen of charged layered oxides can attack carbonate solvents and catalyze electrolyte decomposition ${ }^{42,46}$. It can therefore be concluded that the polyanionic $\left(\mathrm{SO}_{4}\right)^{2-}$ species could stabilize the surface of $\mathrm{Li}_{2} \mathrm{MnO}_{3}$ not only by preventing gas releasing in solution, but also by inhibiting reactions with the electrolyte. The mechanism of prevention of polyanionic $\left(\mathrm{SO}_{4}\right)^{2-}$ against reaction with EC molecule is analyzed below.

To clarify the electronic structure of S deposition, we analyzed the Bader charge during the process of deposition and delithiation, and calculated the LDOS using the HSE06 hybrid functional (Fig. 4a, b). The Bader analysis shows an obvious oxidation of $\mathrm{S}$ ions and a slight reduction of $\mathrm{O}_{\text {sur }}$ upon sulfur deposition (Fig. 4a). This inductive effect with charge transfer from $\mathrm{S}$ to $\mathrm{O}_{\text {sur }}$ facilitates the formation of oxysulfide $\left(\mathrm{SO}_{n}\right)^{m-}$ species. After extraction of $37.5 \% \mathrm{Li}$ ions, the $\left(\mathrm{SO}_{n}\right)^{m-}$ species are further oxidized to polyanionic $\left(\mathrm{SO}_{4}\right)^{2-}$, whereas the oxidation states of $\mathrm{O}_{\text {sur }}$ and $\mathrm{Mn}$ ions are barely changed, demonstrating that $\mathrm{S}$ ions, instead of $\mathrm{O}_{\text {sur }}$, provide the charge compensation on the surface in the first delithiation process. The calculated LDOS on the (010) surface shows that the orbital of the deposited S ions is hybridized with the first layer of $\mathrm{O}$ ions and lies right below the Fermi level (Fig. 4b). The high energy level of the hybridized S-O orbital suggests a preferential oxidation of $\left(\mathrm{SO}_{n}\right)^{m-}$ species. In the $37.5 \%$ delithiated state, with $\mathrm{S}$ ion loss of electrons, these bonding $\mathrm{S}-\mathrm{O}$ states move down to $-7 \mathrm{eV}$, and the antibonding $\mathrm{S}-\mathrm{O}$ states move to $9 \mathrm{eV}$ above the Fermi level, signifying that the polyanionic $\left(\mathrm{SO}_{4}\right)^{2-}$ is redox-inactive during further delithiation or subsequent electrochemical cycles. The more ionic character of the $\mathrm{S}-\mathrm{O}$ bond in $\left(\mathrm{SO}_{4}\right)^{2-}$ accounts for the large energy difference between the occupied and empty states, thus maintaining surface stability in an electrolyte environment (Fig. 3).

Figure $4 \mathrm{c}$ illustrates the mechanism of oxygen evolution and $\mathrm{O}_{\text {sur }}$ stabilization by polyanion induction. For the clean surface of $\mathrm{LLO}, \mathrm{O}_{\text {sur }}$ release during delithiation is known to arise from its reduced bonding-induced overoxidation, by way of rotation to an $\mathrm{O}-\mathrm{O}$ dimer, based on the requirement of charge compensation (left part of Fig. 4c). While for the deposited sulfur, the hybrid 
a
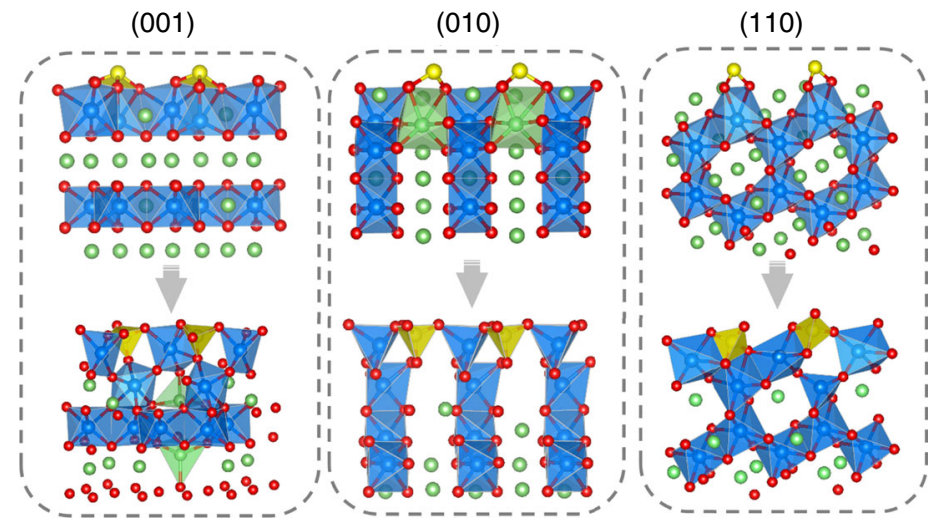

b

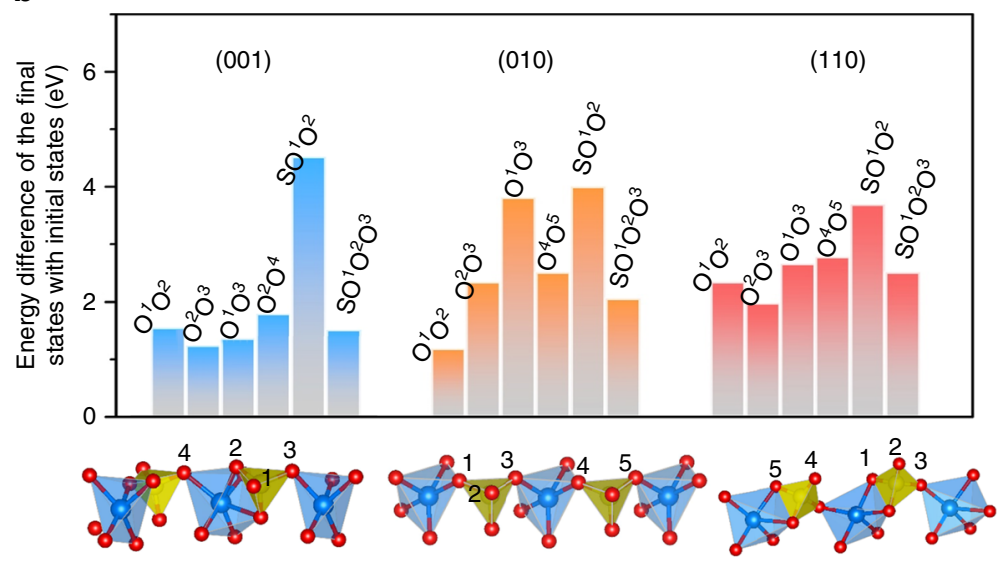

C

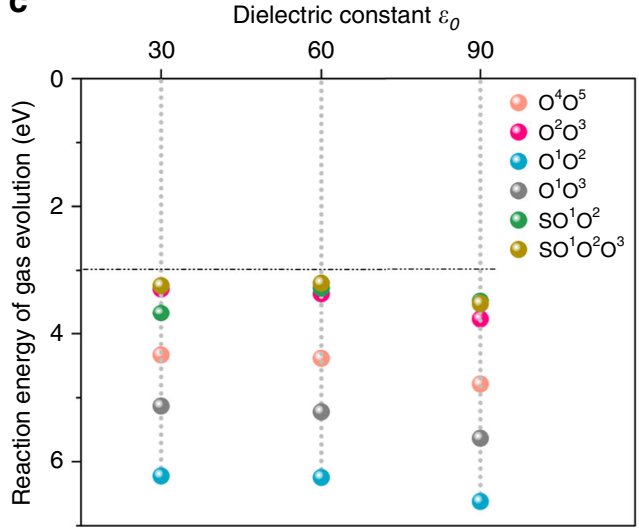

d

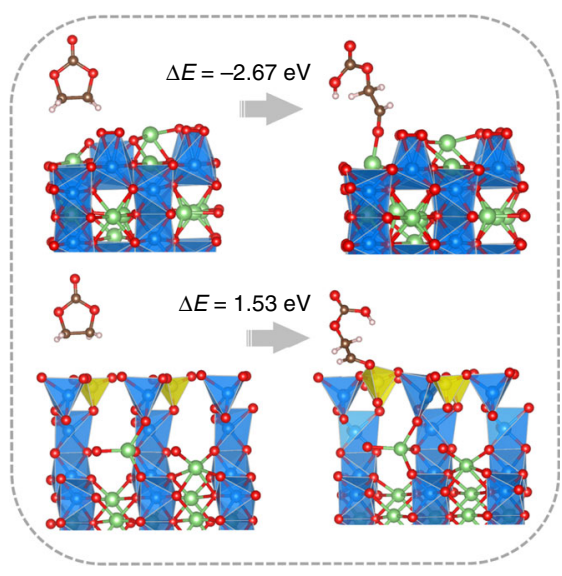

Fig. 3 The surface structures of $\mathrm{Li}_{2} \mathbf{M n O}_{3}$ with sulfur deposition and the stability of surface $\mathbf{O}$ after delithiation. a Structures of the (001), (010), (110) surfaces with sulfur deposition (upper) and the reconstructed configurations upon 37.5\% delithiation (below). The yellow spheres represent $\mathrm{S}$ ions. b Energy required for gas evolution on the three delithiated surfaces. Each exposed $\mathrm{O}$ atom on the surface is labeled with a different number and then is grouped together or with $\mathrm{S}$ atom to form the potential gas products, such as $\mathrm{O}_{2}, \mathrm{SO}_{2}$, or $\mathrm{SO}_{3}$. Each slab with gas evolution to the vacuum is taken as the final state. c Reaction energy of (010) surface oxygen evolution in implicit solvents with different dielectric constants. $\mathbf{d}$ EC molecular adsorption on delithiated (010) surface before and after surface treatment and ring opening of cyclic carbonate.

$\mathrm{S}-\mathrm{O}$ bond enables charge transfer from $\mathrm{S}$ to $\mathrm{O}_{\text {sur }}$, thus preventing the oxidation and rotation of $\mathrm{O}_{\text {sur }}$ to form $\mathrm{O}-\mathrm{O}$ dimers (right part of Fig. 4c). The sulfur ions, which transform from oxysulfide $\left(\mathrm{SO}_{n}\right)^{m-}$ to the polyanionic $\left(\mathrm{SO}_{4}\right)^{2-}$ species, provide the surface charge compensation in the first charging process, and also maintain redox stable during the following cycles. The strategy to replace the nonbonding coordination of $\mathrm{O}_{\text {sur }}$ with surface polyanion configuration is not limited to the LLO series of compounds, but to all materials that suffer from surface instability involving $\mathrm{O}_{\text {sur }}$ oxidation.

Experimental verification. Sulfide deposition experiments were subsequently conducted to test our computational predictions. Specifically, sulfide deposition on $\mathrm{Li}_{1.2} \mathrm{Ni}_{0.2} \mathrm{Mn}_{0.6} \mathrm{O}_{2}$ was conducted and characterized as described in the Supplementary Information. The pristine $\mathrm{Li}_{1.2} \mathrm{Ni}_{0.2} \mathrm{Mn}_{0.6} \mathrm{O}_{2}$ and sulfide-deposited samples are labeled as LP, S-50, S-200, and S-500 according to the deposition concentration of S. The morphology and XRD pattern of LP, S-50, S-200, and S-500, as shown in Supplementary Figs. 9 and 10 , suggest that the morphology and phase constitution of $\mathrm{Li}_{1.2} \mathrm{Ni}_{0.2} \mathrm{Mn}_{0.6} \mathrm{O}_{2}$ powders are unchanged upon $\mathrm{S}$ deposition.

XPS measurements were performed to probe the oxidation states on the surface of each sample (Fig. $5 \mathrm{a}$ ). The main peaks were observed near $167.8 \mathrm{eV}$ in the S $2 p$ core spectra of the S-deposited samples, suggesting the formation of $\mathrm{SO}_{x}$ species instead of $\mathrm{S}^{2-}$ or elemental $\mathrm{S}^{47}$. In addition, although $\mathrm{SO}_{3}{ }^{2-}$ was found to be the major component, $\mathrm{SO}_{4}{ }^{2-}$ groups with a lower $\mathrm{S}$ electron density were observed in S-50 and decreased with a higher concentration of $\mathrm{S}$ vapor. This phenomenon is expected to be associated with Li and oxygen vacancies on the surface of LLOs, and will be explored in further work. The $\mathrm{O}$ 1s core spectra are shown in Supplementary Fig. 11, in which the deposition of sulfur alters the oxidation state of oxygen by increasing the content of oxygen from $\mathrm{SO}_{x}$ species, in peaks located near $532 \mathrm{eV}^{48}$.

The charge-discharge curves performed on Li/LP cells (Fig. 5b) are compared with that of S-deposited samples. LP presented the charge capacity of $238 \mathrm{~mA} \mathrm{~h} \mathrm{~g}^{-1}$ in the first cycle accompanied with a first Coulombic efficiency (FCE) of $65.2 \%$. S absorption showed a slight decreased charge capacity, but an increase in FCE ( $85 \%$ for S-500) in the first cycle; considering that the irreversible processes are closely related to the release of oxygen ${ }^{29,49-51}$, the increased FCE in the S-absorbed samples is likely associated with suppressed irreversible oxygen release from the bulk lattice. It is worth mentioning that previous attempts to increase the FCE of LLO generally shorten the plateau length of the oxygen oxidation reaction (at around $4.5 \mathrm{~V}$ ), that is, inhibiting the reversible and irreversible oxygen reaction in the first charge ${ }^{52,53}$. The oxygen reaction plateau lengths in this research are compared (Fig. $5 \mathrm{c}$ ); the slightly higher percentages of charge capacity between 4.5 and $4.8 \mathrm{~V}$ in the S-deposited sample (S-50 and S-200) suggest that the 


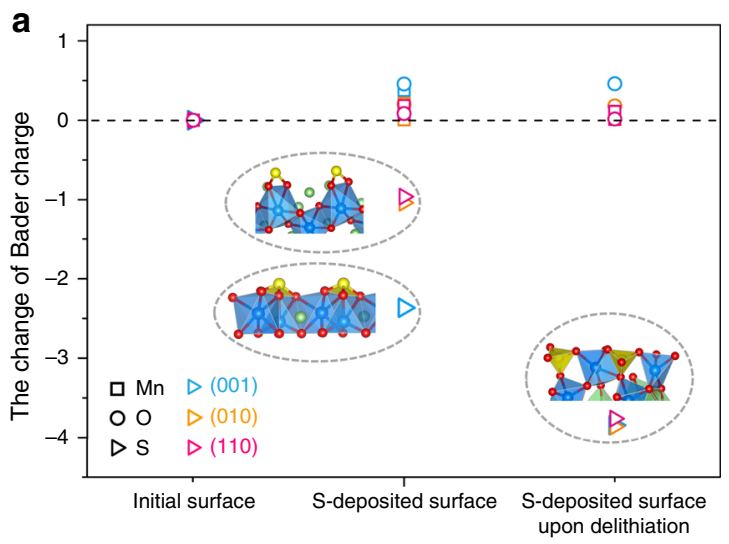

C

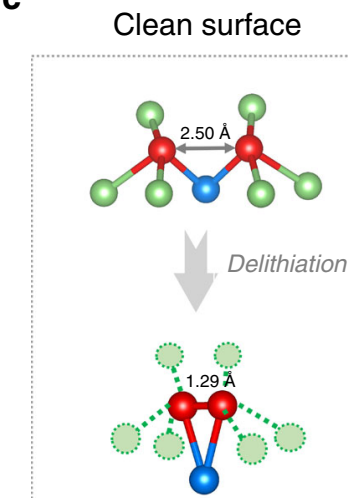

b

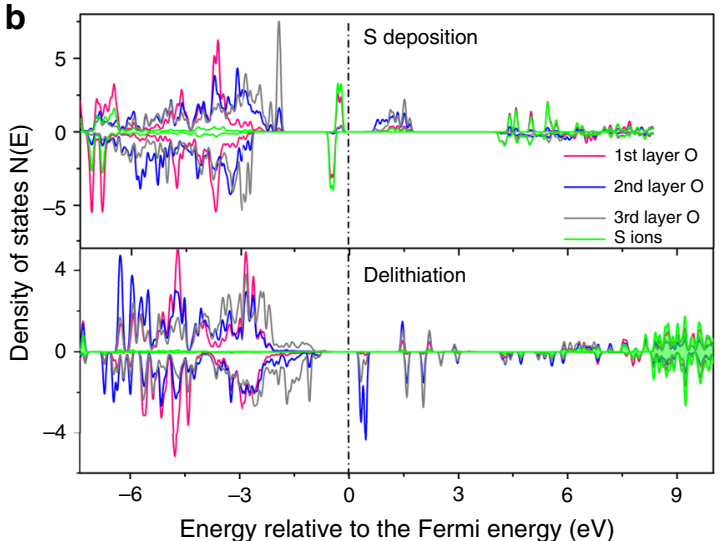

$\mathrm{O}_{\text {sur }}$ oxidization provides charge compensation

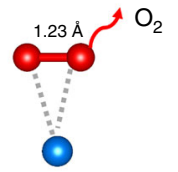

Gaseous $\mathrm{O}_{2}$ evolution from surface

Fig. 4 Surface charge transfer of sulfur-deposited $\mathrm{Li}_{2} \mathbf{M n O}_{3}$ and the mechanism of surface $\mathbf{O}$ stabilization. a The change in Bader charge of surface $\mathrm{Mn}$, $\mathrm{O}$, and $\mathrm{S}$ (per atom) on the three outermost surfaces during the process of $\mathrm{S}$ deposition and latter extraction of $37.5 \%$ Li. Bader charge of surface Mn, $\mathrm{O}$ (per atom) on the initial surface, and $\mathrm{S}$ atoms has been normalized to zero. $\mathbf{b}$ Calculated local density of states of the first layer $\mathrm{O}$ to the third layer $\mathrm{O}$ and $\mathrm{S}$ ions upon $\mathrm{S}$ deposition and $37.5 \%$ delithiation. Here the (010) surface was taken as characteristic of the three surfaces of $\mathrm{Li}_{2} \mathrm{MnO}_{3}$. $\mathbf{c} \mathrm{Schematic}$ of how $\mathrm{S}$ deposition stabilizes surface $\mathrm{O}$ during delithiation in the first cycle (right), by contrast to $\mathrm{O}_{2}$ evolution from the clean surface (left). From top to bottom: the coordination environment of the surface $\mathrm{O}$ ions pairs in full lithiation; the intermediate local structure after $37.5 \%$ delithiation; the final stable configuration of surface $\mathrm{O}$ ions pairs after $37.5 \%$ delithiation.

oxygen redox reaction is maintained. Thus, it can be deduced that only the irreversible oxygen evolution in LLO was suppressed after $\mathrm{S}$ deposition. In addition, the $\mathrm{d} Q / \mathrm{d} V$ curves of the second charge shown in Fig. 5d have enhanced redox activity around $3.0 \mathrm{~V}$ in the S-deposited samples, indicating the formation of a spinel phase after the first cycle. Considering the fact that traditional LLO $\rightarrow$ spinel-phase transitions are fairly slow in the first several cycles 54,55 , the spinel phase in the S-deposited samples seems to be more likely initiated by the $\mathrm{Li} / \mathrm{Mn}$ antisite mixing that is driven by the formation of $\mathrm{SO}_{x}$.

The normalized galvanostatic discharge profiles of LP and S200 obtained at $1 \mathrm{C}$ (Fig. 5e, f) are indicative of the suppressed voltage decay in S-deposited samples. As the voltage decay in LLO is induced by the migration of Mn through oxygen vacancies ${ }^{50,56}$, the suppressed voltage decay in S-200 benefits from the inhibition of irreversible oxygen evolution. The cyclic performance at a 1-C rate and the capacity retention at different rates in Fig. 5g show the enhanced electrochemical stability and rate performance of the S-deposited samples. Specifically, the S-200 sample with the best cyclic stability possesses a capacity retention of $99.0 \%$ (194 $\left.\mathrm{mA} \mathrm{h}^{-1}\right)$ after 100 cycles at $1 \mathrm{C}\left(1 \mathrm{C}=200 \mathrm{~mA} \mathrm{~g}^{-1}\right)$, while the capacity retention is only $70.4 \%\left(121 \mathrm{~mA} \mathrm{~h} \mathrm{~g}^{-1}\right)$ for untreated LP. The specific energy-density retention (Fig. 5h), which is the primary bottleneck of LLO that is influenced by the effects of both voltage and capacity decay, has been significantly improved from $59.5 \%$ to $96.0 \%$ with an optimal amount of S deposition.
The high-angle annular dark-field scanning transmission electron microscopy (HAADF-STEM) technique was used to reveal the atomic structure evolution during de-/lithiation. Prior to electrochemical de-/lithiation, LP showed a typical layered structure on both the interior and the surface region (Supplementary Fig. 12a-c), while the surface of S-200 $(2-3 \mathrm{~nm})$ has a different atomic configuration than that of the interior area (Fig. 6a-e). Compared with the layered features from the projection of the $\langle 010\rangle$ zone axis of the $C 2 / \mathrm{m}$ structure in the interior area (Fig. 6b), TM ions were detected in both the tetrahedral and octahedral sites of the Li layer $\left(\mathrm{O}_{\mathrm{Li}}\right)$ near the surface (Fig. $6 \mathrm{~d})^{50,57,58}$. Such a surface structure, as presented in the atomic models (Fig. 6e), could be considered as a cationmixed $\mathrm{Mn}_{3} \mathrm{O}_{4}$-type structure ${ }^{59-62}$, which is likely due to $\mathrm{S}$ deposition-induced Li leaching and subsequent TM ion migration, as predicted by our DFT calculations. The lowered delithiation voltage (by $1.6 \mathrm{~V}$ as compared with the bulk ${ }^{15}$ ) in the S-deposited surface suggests that Li ions are easily leached out from the surface during the heat treatment. The structural change after partial delithiation (Fig. 3a) clearly shows Mn migration to the Li layer. Therefore, although only $\mathrm{O}$ ions in the outmost surface will bond with $\mathrm{S}$ to form $\mathrm{SO}_{x}{ }^{2-}$ species, based on our DFT calculations, the S-deposition-induced structural evolution could expand into the near-surface region $(2-3 \mathrm{~nm})$. After 100 cycles, 2-3-nm-thick surface reconstruction layer with a different crystal structure was formed on S-200 (Fig. 6f) in the fully discharged 
a

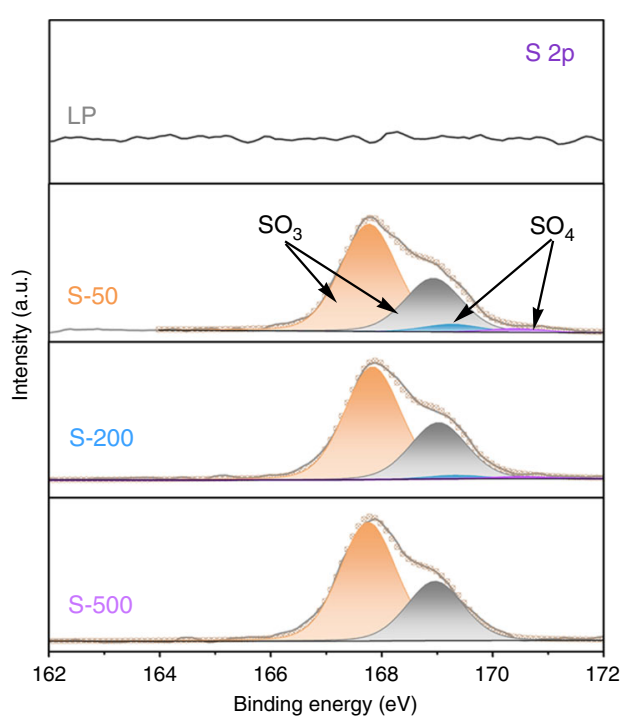

b

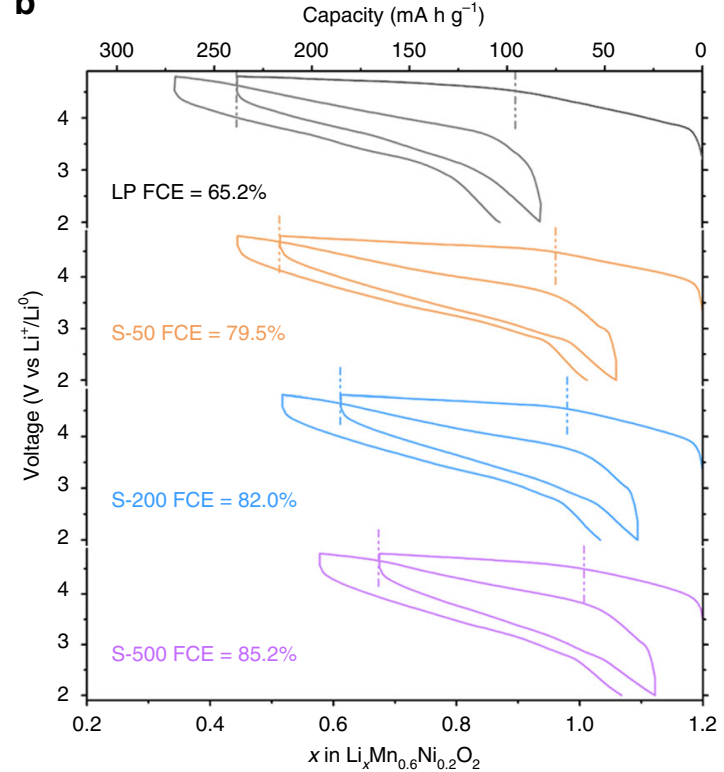

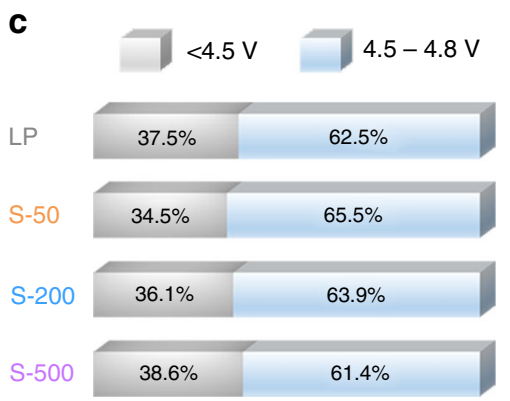
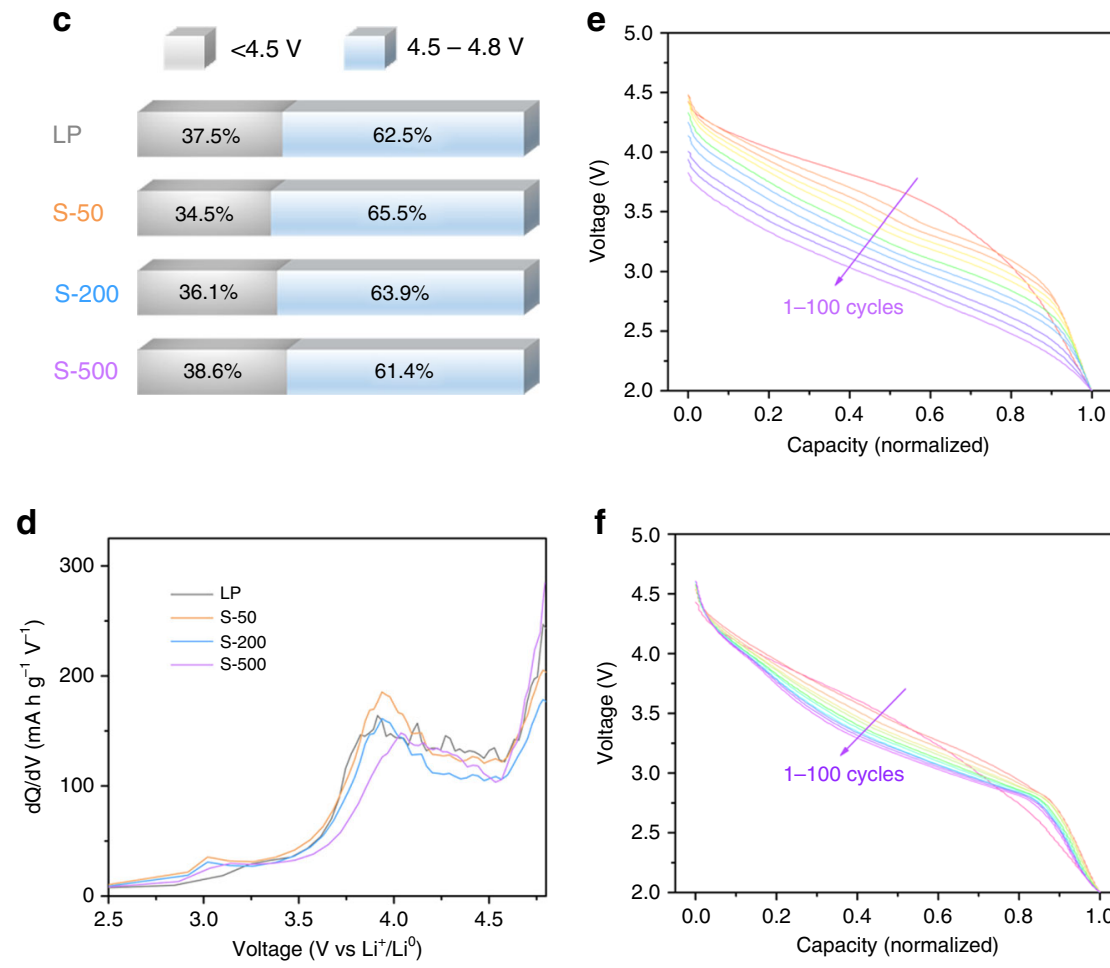

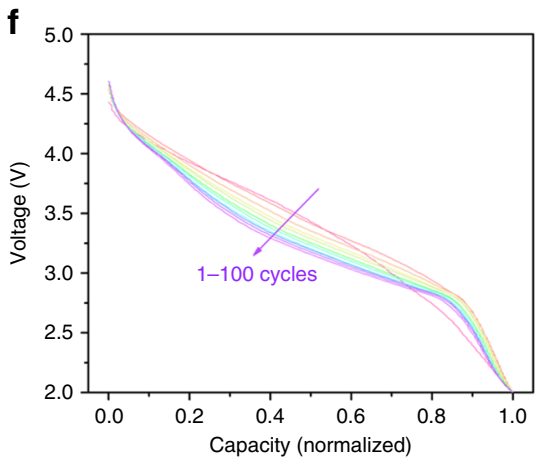

Fig. 5 The surface states and electrochemical behavior of products before and after $\mathbf{S}$ deposition. a $S 2 p$ spectra of products before and after $\mathrm{S}$ deposition. $\mathbf{b}$ Voltage profiles of the first two cycles obtained at $0.5 \mathrm{C}$. Regions from 4.5 to $4.8 \mathrm{~V}$ are marked with vertical dash lines. $\mathbf{c}$ Percentage-stacked bar chart of charge capacities in the first cycle obtained at $0.5 \mathrm{C}$; the total capacities are divided into two sections: $<4.5 \mathrm{~V}$ and $4.5-4.8 \mathrm{~V}$. d dQ/dV curves of the charge process in the second cycle obtained at $0.5 \mathrm{C}$. e Normalized galvanostatic discharge profiles of LP obtained at $1 \mathrm{C}$. $\mathbf{f}$ Normalized galvanostatic discharge profiles of S-200 obtained at 1 C. $\mathbf{g}$ Cyclic performance at $1 \mathrm{C}$ together with the capacity retention at different rates (insets). $\mathbf{h}$ Specific energydensity retention after 100 cycles at $1 \mathrm{C}$.

state. In contrast to the layered configuration in the interior (Fig. 6g), the outmost region appears to be a $\mathrm{LiMn}_{2} \mathrm{O}_{4}$-type spinel structure with a three-dimensional framework constructed by $\mathrm{MO}_{6}$ octahedra (Fig. 6i, j). Note that this $\mathrm{LiMn}_{2} \mathrm{O}_{4}$-type layer is different from the $\mathrm{Mn}_{3} \mathrm{O}_{4}$-type structure seen before cycling, as the TM ions are located in octahedral sites. Such a structural evolution is likely from the further rearrangements of surface atomic configurations upon charging/discharging. However, for the cycled LP electrode, the atomic configuration appears to be primarily composed of a cation-disordered rock-salt structure (Supplementary Fig. 12d-f). This difference is in agreement with the ex situ XRD measurement (Supplementary Fig. 13), which shows a well-preserved phase constitution in cycled S-200, but a much more secondary phase in the cycled LP. In addition, the cycled LP showed an amorphous feature layer with a thickness of 3-4 nm, which is more likely associated with the loss of crystallinity after accumulated oxygen loss, rearrangement of transition-metal ions, and the corrosion of the electrolyte ${ }^{56-60}$. 

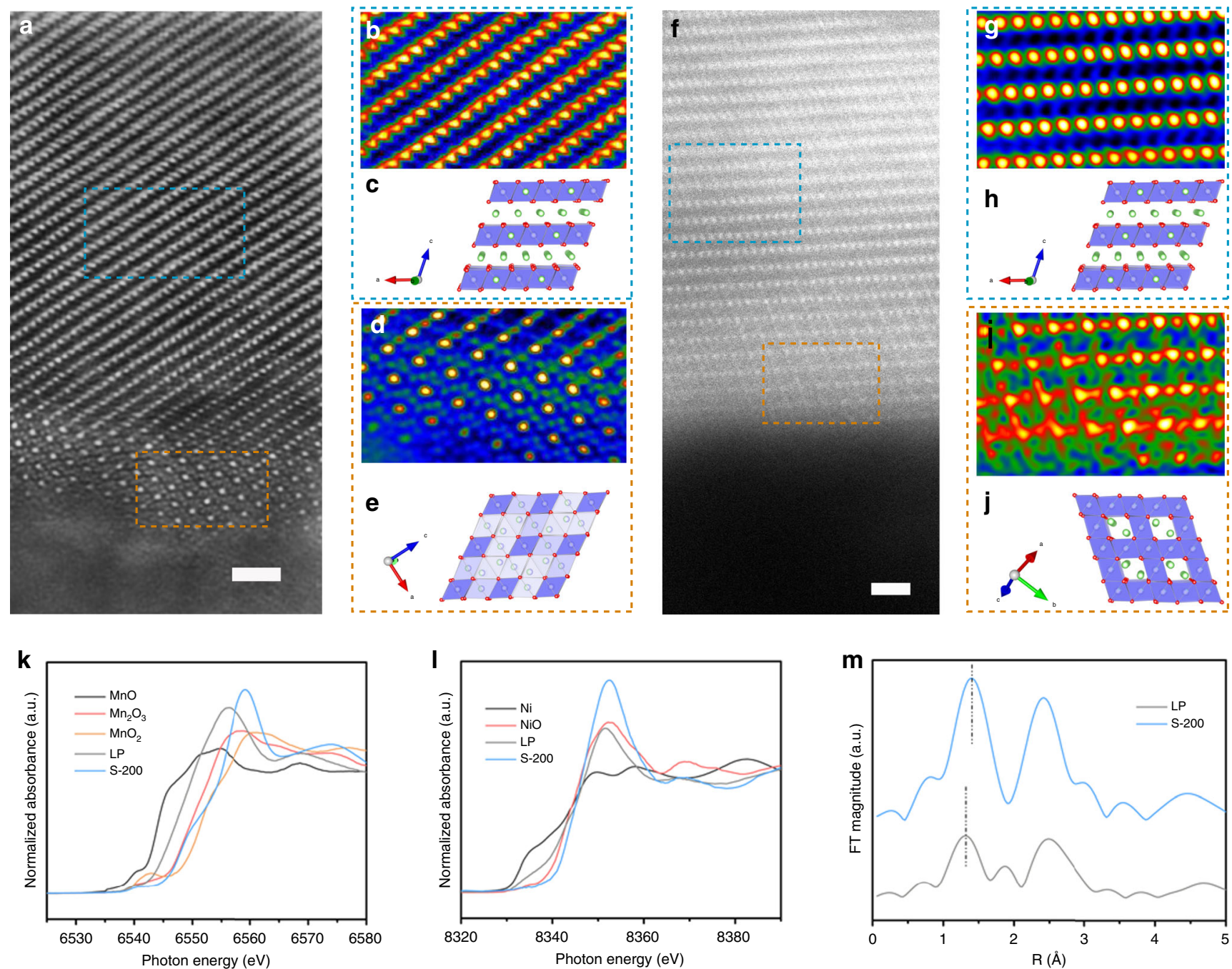

Fig. 6 The atomic structural evolution and oxidation-state change of samples before and after cycling. a-e Atomic configuration of the S-200 before cycling. The overview of HAADF-STEM image (a) shows the crystal structure from surface to the bulk of S-200; the blue and orange dash square showed two regions with different atomic configurations. The interior region marked by the blue dashed square in (a) has been FFT-filtered and shown in (b), and the corresponding atomic model is shown in (c). The spots with higher contrast in (b) show TM ions with higher Z than Li ions; the Li, M, and O ions have been shown as green, blue, and red spheres in (c), respectively. The surface region with cation-mixed $\mathrm{Mn}_{3} \mathrm{O}_{4}$ feature marked by orange dash square in (a) has been FFT-filtered and shown in (d), and the corresponding atomic model has been drawn (e). $\mathbf{f}-\mathbf{j}$ The atomic configuration of S-200 electrode after 100 cycles. The HAADF-STEM image from the surface to the bulk of charged S-200 (f) shows the different atomic configurations on the surface area; the blue and orange dashed square shows two regions with different atomic configurations. The layered feature region marked by the blue dashed square in (f) has been FFT-filtered as shown in ( $\mathbf{g}$ ), and the corresponding atomic model is shown in (h). The region with a spinel feature that is marked by an orange dashed square in (f) has been FFT-filtered and shown in (i), and the corresponding atomic model is shown in (j). The scale bar donates $1 \mathrm{~nm}$. $\mathbf{k}$ Normalized Mn K-edge XANES spectra of cycled LP and S-200. The valence states of Mn were determined by comparison with the spectra of pure-phase MnO, $\mathrm{Mn}_{2} \mathrm{O}_{3}$, and $\mathrm{MnO}_{2}$. I Normalized Ni K-edge XANES spectra of cycled LP and S-200. The valence states of Ni were determined by comparison against the spectra of pure-phase $\mathrm{Ni}$ and $\mathrm{NiO}$. $\mathbf{m}$ The $k^{3}$-weighted Fourier transform magnitudes of Mn K-edge EXAFS spectra obtained from cycled LP and S-200.

For the cycled S-200 (Fig. $6 \mathrm{f}$ ), only the outmost surface ( $\leq 1 \mathrm{~nm}$ ) showed less ordering than that of the interior, suggesting a wellpreserved crystal structure on the surface of cycled S-200. As indicated in previous studies ${ }^{50,56}$, the phase transition of LLO during long-term cycling follows a sequence of layered $\rightarrow$ spinel $\rightarrow$ rock salt. Therefore, we can conclude that the irreversible phase transition has been effectively suppressed in the S-deposited samples.

The anion oxidation behavior of LP and S-200 has also been investigated through ex situ XPS and X-ray absorption near-edge structure/extended X-ray absorption fine structure (XANES/ EXAFS) measurements. For the XPS tests, all cycled electrodes were etched by Ar for $30 \mathrm{~s}$ under an etch voltage of $500 \mathrm{~V}$ to remove the surface species. Although the signal of lattice oxygen is influenced by the shielding effect of surface impurities in the XPS spectra, the O $1 \mathrm{~s}$ spectra of S-200 shows better reversible anion oxidation (well-preserved peroxo-like $\mathrm{O}_{2}^{2-}$ peak located around $530.5 \mathrm{eV}$ ) than that of LP upon long-term cycling (Supplementary Figs. 14 and 15). The S 2p spectra obtained from the S-200 electrodes in the first cycle (Supplementary Fig. 16) suggest the oxidation state of $\mathrm{S}$ to be $\mathrm{SO}_{4}{ }^{2-}$, implying the oxidation from $\mathrm{SO}_{3}{ }^{2-}$ to $\mathrm{SO}_{4}{ }^{2-}$ during the delithiation process $(\leq 4.4 \mathrm{~V})$. Within the de-/lithiation processes in the first cycle, the oxidation state of $\mathrm{S}$ remains at $\mathrm{SO}_{4}{ }^{2-}$, showing that both the $\mathrm{S}$ and the coordinated $\mathrm{O}$ ions in the outmost surface have not participated in charge compensation. After 100 cycles, even the $\mathrm{S}$ $2 p$ peak in the long-term cycled S-200 showed a lower valence state of $\mathrm{S}(\sim 168.5 \mathrm{eV})$, which is likely associated with the small 
amount of $\mathrm{O}$ dissolution or a side reaction between $\mathrm{SO}_{4}{ }^{2-}$ and the SEI/electrolyte; the oxidation state could still be represented as $\mathrm{SO}_{x}{ }^{2-}(3<x<4)$. Therefore, we can conclude that the inductive effect of the $\mathrm{S}-\mathrm{O}$ bond is stable upon long-term cycling. The XAENS spectra of $\mathrm{Mn}$ and $\mathrm{Ni}$ obtained from the pristine sample (Supplementary Fig. 17) show a similar valence state of the TM ions in LP and S-200, indicating that the S adsorption has little influence to the average valence state of the TM ions. However, from the XANES spectra of $\mathrm{Mn}$ and $\mathrm{Ni}$, and EXAFS spectra of $\mathrm{Mn}$ obtained from the cycled electrodes (Fig. $6 \mathrm{k}-\mathrm{m}$ ), both the valence state and atomic configuration vary between LP and S200. From the Mn K-edge spectra (Fig. 6k, l), the average valence states of $\mathrm{Mn}$ ions in cycled LP are between $\mathrm{Mn}^{2+}$ and $\mathrm{Mn}^{3+}$ by comparing with the selected $\mathrm{Mn}$ references, while the S-200 shows a near +4 valence state of $\mathrm{Mn}$ ions. As the ideal valence state of Mn in LLO in the discharged state is +4 , the low valence state of $\mathrm{Mn}$ in cycled LP indicates an irreversible change of $\mathrm{Mn}$ electronic structure upon cycling. Analogously, the Ni K-edge spectra showed a lower valence state of $\mathrm{Ni}$ in cycled LP, while the $\mathrm{Ni}$ valence state is $\sim+2$ in cycled S-200. Therefore, it can be deduced that the transition-metal ions in cycled LP were significantly reduced, which is more likely to be associated with the irreversible processes. According to the Fourier transformed EXAFS spectra of Mn (Fig. $6 \mathrm{~m}$ ), the first coordination peak (at $\sim 1.3 \mathrm{~A}(\mathrm{o})$ ), which is related to the $\mathrm{Mn}-\mathrm{O}$ scattering path in the first shell, showed a shorter $\mathrm{Mn}-\mathrm{O}$ bond in cycled LP than that in S-200, implying more significant distortion of $\mathrm{MnO}_{6}$ in cycled LP. On the basis of previous investigations ${ }^{56,63,64}$, it can be inferred that the shortened $\mathrm{Mn}-\mathrm{O}$ bond in cycled LLO is either contributing to the formation of $\mathrm{MnO}_{4}$ tetrahedral or $\mathrm{MnO}_{5}$ square pyramidal ligands after the formation of oxygen vacancies in $\mathrm{MnO}_{6}$; both of these changes imply irreversible oxygen release upon cycling. Overall, the ex situ XPS and XAS measurements showed better-preserved anion redox as well as a diminished atomic and oxidation- state change in the S-deposited sample. The HAADF/STEM and ex situ XRD results confirm the reduced irreversible phase transition in cycled S-200. Therefore, we can then conclude that the irreversible phase transition has been effectively suppressed in the S-deposited samples.

\section{Discussion}

DFT calculations were performed to investigate the mechanism of surface oxygen evolution in $\mathrm{Li}_{2} \mathrm{MnO}_{3}$ and the surface reconstruction kinetics during delithiation. The calculated LDOS and Bader charges demonstrate a preferential oxidation of $\mathrm{O}_{\text {sur }}$ during delithiation. This was found to be associated with the undercoordinated configuration of $\mathrm{O}_{\text {sur }}$ with cationic vacancies on the surface, which creates an additional O-2p lone-pair $\left(\mathrm{IO}_{2 p}\right)$ orbital with a higher energy level than $\left(\mathrm{IO}_{2 p}\right)$ in $\mathrm{O}_{\text {int }}$. Moreover, the calculated MEP results show that the kinetically feasible $\mathrm{O}_{\text {sur }}$ release gives rise to surface $\mathrm{Mn}$ migration and thus triggers a spinel-like phase transformation. The large reverse barrier for $\mathrm{O}_{2}$ dissociation and $\mathrm{Mn}$ extraction from the spinel-like phase during discharge gives rise to the electrochemical irreversibility. To mitigate $\mathrm{O}_{2}$ release, we introduced less electronegative sulfur into the $\mathrm{Li}_{2} \mathrm{MnO}_{3}$ surface to bond with $\mathrm{O}_{\text {sur. }}$. Our calculations show that sulfur can be stably deposited onto the surface to form oxysulfide $\left(\mathrm{SO}_{3}\right)^{x-}$ and $\left(\mathrm{SO}_{2}\right)^{y-}$ species, which are then further oxidized to stable polyanionic species $\left(\mathrm{SO}_{4}\right)^{2-}$ upon delithiation. This oxidation of sulfur ions provides charge compensation on the surface in the first charge cycle, and thus prevents surface gas release. Accordingly, experimental attempts at surface sulfur deposition were carried out on $\mathrm{Li}_{1.2} \mathrm{Ni}_{0.2} \mathrm{Mn}_{0.6} \mathrm{O}_{2}$, and it was found that $\mathrm{SO}_{x}$ species were formed on the surface of the Streated samples. The sulfur-deposited samples showed significantly reduced irreversible capacity loss in the first cycle $(\mathrm{FCE}=82 \%)$ and superior cyclic stability $(99.0 \%$ capacity and $96.0 \%$ energy density retained) upon 100 cycles at $1 \mathrm{C}$. The combination of ex situ XPS, XRD, and HAADF-STEM measurements shows a reversible anion oxidation and highly conserved crystal structure of the cycled sulfur-deposited samples, indicating the enhanced stability after sulfur deposition. The proposed surface polyanion stabilizing the surface structure can likely be generalized to materials that suffered from instability involving anion oxidation.

\section{Methods}

Computations. All calculations were performed with density functional theory (DFT) as implemented in the Vienna ab initio simulation package (VASP) ${ }^{65}$. The plane-wave basis set and the projector-augmented wave framework were used to describe the valence and core electrons. The electron exchange-correlation energy was evaluated by the Perdew-Burke-Ernzerhof (PBE) functional with a Hubbard $U$ correction $^{66}$. The effective $\mathrm{U}$ value with $4 \mathrm{eV}(\mathrm{J}=1 \mathrm{eV})$ of $\mathrm{Mn}$ was adopted from previous work. The energy cutoff of the plane-wave basis set was $520 \mathrm{eV}$. The Monkhorst-Pack method with a k-point mesh of $7 \times 7 \times 7$ and $3 \times 3 \times 1$ for the bulk and $2 \times 2$ slab models calculations. Cell parameters and atomic positions were full relaxed for bulk optimization. The surface models were built after cleavage of the optimized bulk $\mathrm{Li}_{2} \mathrm{MnO}_{3}$ at the studied orientation. All slabs contain nine to sixteen layers of atoms, depending on different surfaces and intention, of which, the atoms in the bottom three or four layers are fixed with coordinates while other layers are fully relaxed. Sufficient vacuum thickness with $17 \AA$ was contained in each slab to screen the interaction between the slab surfaces. Dipole correction, which is used for balancing net dipole on the surface, was considered in all slab calculations to preserve consistency. Each slab was optimized until the force per atom was less than $0.01 \mathrm{eV} / \AA$.

To accurately investigate the oxygen oxidation activity,

Heyd-Scuseria-Ernzerhof (HSE06) hybrid functional was applied to obtain the density of states. Strongly constrained and appropriately normed density functional $(\mathrm{SCAN})^{33}$ was used to correct energy of oxygen evolution. Basin-hopping algorithm $^{67}$, as implemented in the atomistic simulation environment ${ }^{68}$, was employed to search for the global minimum configurations of the slab in different delithitaion states. In total, 80 or more than $100 \mathrm{Li}$-vacancy arrangements in each delithiated state were examined. Activation barriers for Li diffusion and the reaction of $\mathrm{O}_{2}$ evolution and $\mathrm{Mn}$ migration were obtained using the climbing nudged elastic band method (cNEB) ${ }^{69}$. For the sulfur deposition investigation, the deposition sites on the surface were probed by placing $\mathrm{S}$ atom on different sites and comparing the calculated deposition energy $E_{\mathrm{dep}}$. The deposition energy $E_{\mathrm{dep}}$ was calculated as

$$
E_{\mathrm{dep}}=E_{\mathrm{slab}+\mathrm{S}}^{\mathrm{hkl}}-E_{\mathrm{slab}}^{\mathrm{hkl}}-E_{\mathrm{S}}
$$

where $E_{\text {slab }+S}^{\mathrm{hkl}}$ is the energy of the slab with deposited S, $E_{\text {slab }}^{\text {hkl }}$ is the energy of the clean surface, and $E_{\mathrm{S}}$ is the energy of the elemental sulfur, which is referred to Materials project ${ }^{70}$. The gas-evolution behavior in the presence of solvent was investigated using an implicit solvent model as implemented in the VASPsol package ${ }^{38}$. The reaction between surface and ethylene carbonate (EC) molecule was explored through EC ring-opening reaction after adsorption on delithiated surface. The reaction energy $\Delta E$ was calculated as

$$
\Delta E=E_{\text {slab }+\mathrm{EC}}-E_{\text {slab }}-E_{\mathrm{EC}},
$$

where $E_{\text {slab }+\mathrm{EC}}$ is the energy of the slab after EC molecule adsorption, $E_{\text {slab }}$ is the energy of the surface before EC adsorption, and $E_{\mathrm{EC}}$ is the energy of EC molecule. All energies were corrected by the SCAN functional.

Synthesis of $\mathbf{L i}_{\mathbf{1 . 2}} \mathbf{N i}_{\mathbf{0 . 2}} \mathbf{M n}_{\mathbf{0 . 6}} \mathbf{O}_{\mathbf{2}} \cdot \mathrm{Li}_{1.2} \mathrm{Ni}_{0.2} \mathrm{Mn}_{0.6} \mathrm{O}_{2}$ was synthesized through coprecipitation method. Lithium hydroxide monohydrate $\left(\mathrm{LiOH} \cdot \mathrm{H}_{2} \mathrm{O}\right)$, manganese sulfate monohydrate $\left(\mathrm{MnSO}_{4} \cdot \mathrm{H}_{2} \mathrm{O}\right)$, nickel sulfate hexahydrate $\left(\mathrm{NiSO}_{4} \cdot 6 \mathrm{H}_{2} \mathrm{O}\right)$, and sodium carbonate $\left(\mathrm{Na}_{2} \mathrm{CO}_{3}\right)$ were purchased from Sigma-Aldrich and used without further purification. A $0.8 \mathrm{M}$ aqueous solution of transition metals was obtained by dissolving $\mathrm{MnSO}_{4} \cdot \mathrm{H}_{2} \mathrm{O}$ and $\mathrm{NiSO}_{4} \cdot 6 \mathrm{H}_{2} \mathrm{O}$ with the $\mathrm{Mn}$ :Ni molar ratio of 3:1 into 10 $\mathrm{ml}$ of deionized water. In total, $1 \mathrm{~g}$ of $\mathrm{Na}_{2} \mathrm{CO}_{3}$ was added into the mixture of $10 \mathrm{ml}$ of ethanol and $20 \mathrm{ml}$ of deionized water to form the precipitating agent; the solution was stirred until $\mathrm{Na}_{2} \mathrm{CO}_{3}$ was completely dissolved. The precipitating agent was added into the solution of transition metals drop by drop under continuous stirring; the solution was kept with stirring for $1 \mathrm{~h}$ and then washed by deionized water and ethanol before drying at $80^{\circ} \mathrm{C}$. The obtained precursor was mixed with $\mathrm{LiOH}$ with a molar ratio of $\mathrm{Li}: \mathrm{M}(\mathrm{M}=\mathrm{Mn}+\mathrm{Ni})=1.65: 1$ and then heated to $800^{\circ} \mathrm{C}$ for $12 \mathrm{~h}$ with a ramping rate of $3.5^{\circ} \mathrm{C} / \mathrm{min}$. The products were washed with deionized water and ethanol and finally dried in an oven at $60^{\circ} \mathrm{C}$ for $4 \mathrm{~h}$. The obtained pristine $\mathrm{Li}_{1.2} \mathrm{Ni}_{0.2} \mathrm{Mn}_{0.6} \mathrm{O}_{2}$ powders were labeled as LP.

Deposition of sulfur. In total, $40 \mathrm{mg}$ of as-prepared $\mathrm{Li}_{1.2} \mathrm{Ni}_{0.2} \mathrm{Mn}_{0.6} \mathrm{O}_{2}$ powder and different amount of sulfur powder (Aldrich) were placed in two crucibles separately 
before transferring into tube furnace; the tube was evacuated and sealed to avoid the oxidation of sulfur. To create the sulfur atmosphere and promote the sulfur absorption, the furnace was heated to $250^{\circ} \mathrm{C}$ with the ramping rate of $15^{\circ} \mathrm{C} / \mathrm{min}$ and maintained for $20 \mathrm{~min}$ until naturally cooled. Further vacuum annealing was carried out for the obtained products at $250^{\circ} \mathrm{C}$ for $20 \mathrm{~min}$ to remove the residual elemental sulfide. Different amount of sulfide was used to make sulfur atmosphere with various concentrations; samples exposed in sulfur atmosphere with the sulfur resource of 50, 200, and $500 \mathrm{mg}$ were labeled as S-50, S-200, and S-500, respectively. All the S-deposited samples were stored into Ar-filled glovebox directly after the $\mathrm{S}$ deposition.

Characterization. The phase constitution was investigated by XRD patterns that were performed on PANalytical-Empyrean XRD with $\mathrm{Cu} \mathrm{K} \alpha$ radiation $(\lambda=$ $0.15405 \mathrm{~nm}, 40 \mathrm{kV}, 40 \mathrm{~mA}$ ). SEM observation was carried out on a FEI Sirion XL30 SEM.

HAADF-STEM. For the preparation of HAADF-STEM samples before cycling, samples were first grinded in a mortar and then dispersed in ethanol, the solution was sonicated for $30 \mathrm{~min}$, and the supernatant was deposited onto holey carbon grids. For the preparation of HAADF-STEM samples after cycling, all the processes were handled in Ar-filled glovebox. The cycled batteries were dissembled, and the electrodes were first immersed in dimethyl carbonate for $24 \mathrm{~h}$; further rinses were carried out by washing the electrodes with dimethyl carbonate five times to remove the residual electrolytes. Active materials were scraped from the electrodes and crushed in the mortar in anhydrous hexane before depositing onto holey carbon grids. The specimens were sealed in Ar-filled container and stored in a glovebox until transferring into a microscope column. The HAADF-STEM images were obtained on a double-aberration-corrected S/TEM (FEI Titan Cubed G2 60-300) operated at $300 \mathrm{kV}$.

X-ray spectroscopy characterization. XPS measurements were performed on a Thermo Fisher Scientific ESCALab 250Xi. The cycled batteries were dissembled and washed in a glovebox; the electrodes were immersed in dimethyl carbonate for $24 \mathrm{~h}$ and further washed by dimethyl carbonate five times to remove the residual electrolytes. The samples were dispersed onto the sample holders and then sealed into a homemade protector inside a glovebox, and the protector was stored in the glovebox before transferring into the XPS chamber. For the ex situ XPS measurements, to eliminate the shielding effect of residual electrolyte and solid electrolyte interphase (SEI) layer on the surface, all electrodes were etched by Ar for $30 \mathrm{~s}$ under an etch voltage of $500 \mathrm{~V}$. The calibration of all the spectra is carried out with the $\mathrm{C} 1 \mathrm{~s}$ peak position at $284.8 \mathrm{eV}$. Each fitting was carried out with a minimum number of components involved, and error bars were calculated by adopting the method in ref. ${ }^{20}$, which probes the XPS detection limitation by fitting pristine samples with three and four peaks. XANES and EXAFS were carried out at the Canadian Light Source Inc.; all XAS data were processed using Athena program.

Electrochemical characterization. The samples were mixed with Super $\mathrm{P}$ and poly (vinylidene fluoride) (PVDF) with the weight ratio of 7.5:1.5:1. N-methyl-2 pyrrolidone (NMP) was added into the mixture until a uniform slurry was formed; the slurry was spread onto $\mathrm{Al}$ foil and dried at $60^{\circ} \mathrm{C}$. The foil was punched into square pieces with the typical loading of active materials ranging from 1.0 to $2.0 \mathrm{mg} \mathrm{cm}^{-2}$. The electrodes were further dried in a vacuum oven at $120^{\circ} \mathrm{C}$ for $10 \mathrm{~h}$ before transferring into the glovebox. The electrochemical measurements were performed with coin cells (CR2025), in which lithium foil acted as both counter- and reference electrodes. The electrolyte (CAPCHEM) was composed of $1 \mathrm{M} \mathrm{LiPF}_{6}$ solution in ethylene carbonate (EC)/dimethyl carbonate (DMC) mixture solution (1:1 by volume), and the Celgard 2400 membrane was used as separator. Galvanostatic charge/discharge cycling was tested on CT2001A battery test systems (LAND Wuhan Corp., China) within the voltage range of $2.0-4.8 \mathrm{~V}$ at room temperature.

\section{Data availability}

The data that support the findings of this study are available from the corresponding author upon reasonable request.

Received: 25 October 2019; Accepted: 8 June 2020;

Published online: 08 July 2020

\section{References}

1. Seo, D.-H. et al. The structural and chemical origin of the oxygen redox activity in layered and cation-disordered Li-excess cathode materials. Nat. Chem. 8, 692-697 (2016).

2. Assat, G. \& Tarascon, J.-M. Fundamental understanding and practical challenges of anionic redox activity in Li-ion batteries. Nat. Energy 3, 373-386 (2018).
3. Saubanère, M., McCalla, E., Tarascon, J. M. \& Doublet, M. L. The intriguing question of anionic redox in high-energy density cathodes for Li-ion batteries. Energ. Environ. Sci. 9, 984-991 (2016).

4. Castel, E. et al. Differential electrochemical mass spectrometry study of the interface of $\mathrm{xLi}_{2} \mathrm{MnO}_{3} \cdot(1-\mathrm{x}) \mathrm{LiMO}_{2}(\mathrm{M}=\mathrm{Ni}, \mathrm{Co}$, and $\mathrm{Mn})$ material as a positive electrode in Li-ion batteries. Chem. Mater. 26, 5051-5057 (2014).

5. Denis, Y., Yanagida, K., Kato, Y. \& Nakamura, H. Electrochemical activities in $\mathrm{Li}_{2} \mathrm{MnO}_{3}$. J. Electrochem. Soc. 156, A417-A424 (2009).

6. Dogan, F. et al. Solid state NMR studies of $\mathrm{Li}_{2} \mathrm{MnO}_{3}$ and Li-rich cathode materials: proton insertion, local structure, and voltage fade. J. Electrochem. Soc. 162, A235-A243 (2015).

7. Zheng, J. et al. Li-and Mn-rich cathode materials: challenges to commercialization. Adv. Energy Mater. 7, 1601284 (2017).

8. $\mathrm{Xu}$, J. et al. Elucidating anionic oxygen activity in lithium-rich layered oxides. Nat. Commun. 9, 947 (2018).

9. Radin, M. D., Vinckeviciute, J., Seshadri, R. \& Van der Ven, A. Manganese oxidation as the origin of the anomalous capacity of Mn-containing Li-excess cathode materials. Nat. Energy 4, 639-646 (2019).

10. Xie, Y., Saubanère, M. \& Doublet, M. L. Requirements for reversible extracapacity in Li-rich layered oxides for Li-ion batteries. Energ. Environ. Sci. 10, 266-274 (2017)

11. Gao, Y. et al. Selecting substituent elements for Li-rich mn-based cathode materials by density functional theory (DFT) calculations. Chem. Mater. 27, 3456-3461 (2015)

12. Marusczyk, A. et al. Oxygen activity and peroxide formation as charge compensation mechanisms in $\mathrm{Li}_{2} \mathrm{MnO}_{3}$. J. Mater. Chem. A 5, 15183-15190 (2017).

13. Bercx, M., Slap, L., Partoens, B. \& Lamoen, D. First-principles investigation of the stability of the oxygen framework of Li-rich battery cathodes. MRS Adv. 4, 813-820 (2019).

14. Lee, E. \& Persson, K. A. Structural and chemical evolution of the layered Liexcess $\mathrm{Li}_{\mathrm{x}} \mathrm{MnO}_{3}$ as a function of $\mathrm{Li}$ content from first-principles calculations. Adv. Energy Mater. 4, 1400498 (2014).

15. Hoang, K. Defect physics, delithiation mechanism, and electronic and ionic conduction in layered lithium manganese oxide cathode materials. Phys. Rev. Appl. 3, 024013 (2015).

16. Croy, J. R. et al. First-charge instabilities of layered-layered lithium-ionbattery materials. Phys. Chem. Chem. Phys. 17, 24382-24391 (2015).

17. Koga, H. et al. Different oxygen redox participation for bulk and surface: a possible global explanation for the cycling mechanism of $\mathrm{Li}_{1.20} \mathrm{Mn}_{0.54} \mathrm{Co}_{0.13} \mathrm{Ni}_{0.13} \mathrm{O}_{2}$. J. Power Sources 236, 250-258 (2013).

18. $\mathrm{Li}, \mathrm{B}$. et al. Understanding the stability for Li-rich layered oxide $\mathrm{Li}_{2} \mathrm{RuO}_{3}$ cathode. Adv. Funct. Mater. 26, 1330-1337 (2016).

19. Li, B., Yan, H., Zuo, Y. \& Xia, D. Tuning the reversibility of oxygen redox in lithium-rich layered oxides. Chem. Mater. 29, 2811-2818 (2017).

20. McCalla, E. et al. Visualization of O-O peroxo-like dimers in high-capacity layered oxides for Li-ion batteries. Science 350, 1516-1521 (2015).

21. Chen, Q. et al. Structural transformations in $\mathrm{Li}_{2} \mathrm{MnSiO}_{4}$ : evidence that a $\mathrm{Li}$ intercalation material can reversibly cycle through a disordered phase. J. Mater. Chem. A 5, 16722-16731 (2017).

22. Ning, F. et al. Surface thermodynamic stability of Li-rich $\mathrm{Li}_{2} \mathrm{MnO}_{3}$ : effect of defective graphene. Energy Storage Mater. 22, 113-119 (2019).

23. Qiu, B. et al. Gas-solid interfacial modification of oxygen activity in layered oxide cathodes for lithium-ion batteries. Nat. Commun. 7, 12108 (2016).

24. Shin, Y. et al. Alleviating oxygen evolution from Li-excess oxide materials through theory-guided surface protection. Nat. Commun. 9, 4597 (2018).

25. Pei, Y. et al. Chelate-induced formation of $\mathrm{Li}_{2} \mathrm{MnSiO}_{4}$ nanorods as a high capacity cathode material for Li-ion batteries. J. Mater. Chem. A 4, 9447-9454 (2016).

26. Pei, Y. et al. Reviving reversible anion redox in 3d-transition-metal Li rich oxides by introducing surface defects. Nano Energy 71, 104644 (2020).

27. Kim, D. et al. Understanding of surface redox behaviors of $\mathrm{Li}_{2} \mathrm{MnO}_{3}$ in Li-ion batteries: first-principles prediction and experimental validation. ChemSusChem 8, 3255-3262 (2015).

28. Heyd, J., Scuseria, G. E. \& Ernzerhof, M. Hybrid functionals based on a screened Coulomb potential. J. Chem. Phys. 118, 8207-8215 (2003).

29. $\mathrm{Hu}, \mathrm{E}$. et al. Evolution of redox couples in $\mathrm{Li}-$ and $\mathrm{Mn}$-rich cathode materials and mitigation of voltage fade by reducing oxygen release. Nat. Energy 3 , 690-698 (2018).

30. Xu, B., Fell, C. R., Chi, M. \& Meng, Y. S. Identifying surface structural changes in layered Li-excess nickel manganese oxides in high voltage lithium ion batteries: a joint experimental and theoretical study. Energ. Environ. Sci. 4, 2223 (2011)

31. Ben Yahia, M., Vergnet, J., Saubanere, M. \& Doublet, M. L. Unified picture of anionic redox in Li/Na-ion batteries. Nat. Mater. 18, 496-502 (2019).

32. Causà, $M$. et al. Electron localization function and maximum probability domains analysis of semi-ionic oxides crystals, surfaces and surface defects. Comput. Theor. Chem. 1053, 315-321 (2015). 
33. Sun, J., Ruzsinszky, A. \& Perdew, J. P. Strongly constrained and appropriately normed semilocal density functional. Phys. Rev. Lett. 115, 036402 (2015).

34. Sun, J. et al. Accurate first-principles structures and energies of diversely bonded systems from an efficient density functional. Nat. Chem. 8, 831 (2016).

35. Lian, X. et al. Calculations of oxide formation on low-index $\mathrm{Cu}$ surfaces. J. Chem. Phys. 145, 044711 (2016).

36. Lian, X., Xiao, P., Liu, R. \& Henkelman, G. Calculations of oxygen adsorptioninduced surface reconstruction and oxide formation on $\mathrm{Cu}$ (100). Chem. Mater. 29, 1472-1484 (2017)

37. Melot, B. C. \& Tarascon, J.-M. Design and preparation of materials for advanced electrochemical storage. Acc. Chem. Res. 46, 1226-1238 (2013).

38. Mathew, K. et al. Implicit self-consistent electrolyte model in plane-wave density-functional theory. J. Chem. Phys. 151, 234101 (2019).

39. Stauffer, S. K. \& Vilčiauskas, L. Computational study of chemical and electrochemical intercalation of $\mathrm{Li}$ into $\mathrm{Li}_{1+\mathrm{x}} \mathrm{Ti}_{2} \mathrm{O}_{4}$ spinel structures. J. Phys. Chem. C. 122, 7779-7789 (2018).

40. Yu, L. et al. Preferential adsorption of solvents on the cathode surface of lithium ion batteries. Angew. Chem. Int. Ed. 52, 5753-5756 (2013).

41. Liu, $\mathrm{H}$. et al. Adsorption of propylene carbonate (PC) on the $\mathrm{LiCoO}_{2}$ surface investigated by nonlinear vibrational spectroscopy. J. Phys. Chem. C. 113 20531-20534 (2009).

42. Okuno, Y. et al. Structures, electronic states, and reactions at interfaces between $\mathrm{LiNi}_{0.5} \mathrm{Mn}_{1.5} \mathrm{O}_{4}$ cathode and ethylene carbonate electrolyte: a firstprinciples study. J. Phys. Chem. C. 123, 2267-2277 (2019).

43. Tebbe, J. L., Fuerst, T. F. \& Musgrave, C. B. Degradation of ethylene carbonate electrolytes of lithium ion batteries via ring opening activated by $\mathrm{LiCoO}_{2}$ cathode surfaces and electrolyte species. ACS Appl. Mater. Interfaces $\mathbf{8}$, 26664-26674 (2016).

44. Ohsaki, T. et al. Overcharge reaction of lithium-ion batteries. J. Power Sources 146, 97-100 (2005).

45. Wang, Q. et al. Thermal runaway caused fire and explosion of lithium ion battery. J. Power Sources 208, 210-224 (2012).

46. Gauthier, M. et al. Electrode-electrolyte interface in Li-ion batteries: current understanding and new insights. J. Phys. Chem. Lett. 6, 4653-4672 (2015).

47. Wilde, L. et al. Adsorption and temperature-dependent decomposition of $\mathrm{SO}_{2}$ on $\mathrm{Ni}(110)$ : an XPS and XAFS study. Surf. Sci. 405, 215-227 (1998).

48. Galtayries, A., Cousi, C., Zanna, S. \& Marcus, P. $\mathrm{SO}_{2}$ adsorption at room temperature on $\mathrm{Ni}(111)$ surface studied by XPS. Surf. Interface Anal. 36, 997-1000 (2004).

49. Zheng, J. et al. Functioning mechanism of $\mathrm{AlF}_{3}$ coating on the Li- and $\mathrm{Mn}$ rich cathode materials. Chem. Mater. 26, 6320-6327 (2014).

50. Zheng, J. et al. Structural and chemical evolution of Li- and Mn-rich layered cathode material. Chem. Mater. 27, 1381-1390 (2015).

51. Yan, P. et al. Tailoring grain boundary structures and chemistry of Ni-rich layered cathodes for enhanced cycle stability of lithium-ion batteries. Nat. Energy 3, 600-605 (2018).

52. Ye, D. et al. Capacity-controllable Li-rich cathode materials for lithium-ion batteries. Nano Energy 6, 92-102 (2014).

53. Wang, C.-C., Jarvis, K. A., Ferreira, P. J. \& Manthiram, A. Effect of synthesis conditions on the first charge and reversible capacities of lithium-rich layered oxide cathodes. Chem. Mater. 25, 3267-3275 (2013).

54. Pei, Y. et al. Understanding the phase transitions in spinel-layered-rock salt system: criterion for the rational design of LLO/spinel nanocomposites. Nano Energy 40, 566-575 (2017).

55. Pei, Y. et al. Phase transition induced synthesis of layered/spinel heterostructure with enhanced electrochemical properties. Adv. Funct. Mater. 27, 1604349 (2017)

56. Mohanty, D. et al. Unraveling the voltage-fade mechanism in high-energydensity lithium-ion batteries: origin of the tetrahedral cations for spinel conversion. Chem. Mater. 26, 6272-6280 (2014).

57. Yan, P., Zheng, J., Zhang, J. G. \& Wang, C. Atomic resolution structural and chemical imaging revealing the sequential migration of $\mathrm{Ni}, \mathrm{Co}$, and $\mathrm{Mn}$ upon the battery cycling of layered cathode. Nano Lett. 17, 3946-3951 (2017).

58. Yan, P. et al. Evolution of lattice structure and chemical composition of the surface reconstruction layer in $\mathrm{Li}_{1.2} \mathrm{Ni}_{0.2} \mathrm{Mn}_{0.6} \mathrm{O}_{2}$ cathode material for lithium ion batteries. Nano Lett. 15, 514-522 (2015).

59. Yan, P. et al. Probing the degradation mechanism of $\mathrm{Li}_{2} \mathrm{MnO}_{3}$ cathode for Liion batteries. Chem. Mater. 27, 975-982 (2015)

60. Yan, P. et al. Phosphorus enrichment as a new composition in the solid electrolyte interphase of high-voltage cathodes and its effects on battery cycling. Chem. Mater. 27, 7447-7451 (2015).
61. Amos, C. D. et al. Revealing the reconstructed surface of $\mathrm{Li}\left[\mathrm{Mn}_{2}\right] \mathrm{O}_{4}$. Nano Lett. 16, 2899-2906 (2016).

62. Gao, X. et al. Oxygen loss and surface degradation during electrochemical cycling of lithium-ion battery cathode material $\mathrm{LiMn}_{2} \mathrm{O}_{4}$. J. Mater. Chem. A 7, 8845-8854 (2019).

63. Croy, J. R., Balasubramanian, M., Gallagher, K. G. \& Burrell, A. K. Review of the U.S. Department of Energy's "deep dive" effort to understand voltage fade in Li- and Mn-rich cathodes. Acc. Chem. Res. 48, 2813-2821 (2015).

64. Zhang, K. et al. Voltage decay in layered Li-rich Mn-based cathode materials. Electro Ener. Rev. 2, 606-623 (2019)

65. Kohn, W., Becke, A. D. \& Parr, R. G. Density functional theory of electronic structure. J. Phys. Chem. 100, 12974-12980 (1996).

66. Liechtenstein, A. I., Anisimov, V. I. \& Zaanen, J. Density-functional theory and strong interactions: orbital ordering in Mott-Hubbard insulators. Phys. Rev. B 52, R5467 (1995).

67. Wales, D. J. \& Doye, J. P. Global optimization by basin-hopping and the lowest energy structures of Lennard-Jones clusters containing up to 110 atoms. J. Phys. Chem. A 101, 5111-5116 (1997).

68. Bahn, S. R. \& Jacobsen, K. W. An object-oriented scripting interface to a legacy electronic structure code. Comput. Sci. Eng. 4, 56-66 (2002).

69. Henkelman, G. \& Jónsson, H. Improved tangent estimate in the nudged elastic band method for finding minimum energy paths and saddle points. J. Chem. Phys. 113, 9978-9985 (2000).

70. Jain, A. et al. Commentary: the materials project: a materials genome approach to accelerating materials innovation. APL Mater. 1, 011002 (2013).

\section{Acknowledgements}

This work was supported by the Welch Foundation under grant F-1841. Computational resources were provided by the Texas Advanced Computing Center. Part of P.X.'s work was performed under the auspices of the U.S. Department of Energy by Lawrence Livermore National Laboratory under Contract Number DE-AC52-07NA27344. Q.C. and Y.P. would like to thank the China Scholarship Council for supporting their study as exchange students at the University of Texas at Austin and the University of Washington.

\section{Competing interests}

The authors declare no competing interests.

\section{Additional information}

Supplementary information is available for this paper at https://doi.org/10.1038/s41467020-17126-3.

Correspondence and requests for materials should be addressed to C.-Y.X., P.X. or G.H.

Peer review information Nature Communications thanks Lin $\mathrm{Gu}$, and the other, anonymous, reviewer(s) for their contribution to the peer review of this work.

Reprints and permission information is available at http://www.nature.com/reprints

Publisher's note Springer Nature remains neutral with regard to jurisdictional claims in published maps and institutional affiliations.

\footnotetext{
(c) (i) Open Access This article is licensed under a Creative Commons Attribution 4.0 International License, which permits use, sharing, adaptation, distribution and reproduction in any medium or format, as long as you give appropriate credit to the original author(s) and the source, provide a link to the Creative Commons license, and indicate if changes were made. The images or other third party material in this article are included in the article's Creative Commons license, unless indicated otherwise in a credit line to the material. If material is not included in the article's Creative Commons license and your intended use is not permitted by statutory regulation or exceeds the permitted use, you will need to obtain permission directly from the copyright holder. To view a copy of this license, visit http://creativecommons.org/ licenses/by/4.0/.
}

(C) The Author(s) 2020 